\title{
Current effects on scattering of surface gravity waves by bottom topography
}

\author{
By RUDY MAGNE AND FABRICE ARDHUIN $^{1}$ \\ ${ }^{1}$ Centre Militaire d'Océanographie, Service Hydrographique et Océanographique de la Marine, \\ 29275 Brest, France \\ ${ }^{2}$ Laboratoire de Sondages Electromagnétique de l'Environnement Terrestre, Université de \\ Toulon et du Var, La Garde, France \\ ardhuin@shom.fr
}

(Received 8 September 2005 (draft))

The scattering of random surface gravity waves by topography of small amplitude, and horizontal scales of the order of the wavelength, is investigated theoretically in the presence of a an almost uniform irrotational current. This problem is relevant to ocean waves propagation on shallow continental shelves where tidal currents are often significant. Defining the small scale bottom amplitude normalized by the mean water depth, $\eta=h / H$, a perturbation expansion of the wave action to order $\eta^{2}$ yields an evolution equation for the wave action spectrum. Based on numerical calculations for sinusoidal bars, a mixed surface-bottom bispectrum, that arises at order $\eta$, is unlikely to be significant in most oceanic conditions. Neglecting that term, the present theory yields a closed equation with a scattering source term that gives the rate of exchange of action between spectral wave components that have the same absolute frequency. This source term is proportional to the bottom elevation variance at the resonant wavenumbers, and thus represents a Bragg scattering approximation. With current, the source term formally combines a direct effect of the bottom topography with an indirect effect of the bottom through the modulation of the surface current and mean surface elevation. For Froude numbers of the order of 0.6 or less, the bottom topography effects dominate. For all Froude numbers, the reflection coefficients for the wave amplitudes that are inferred from the source term are asymptotically identical, as $\eta$ goes to zero, to previous theoretical results for monochromatic waves propagating in one dimension over sinusoidal bars. In particular, the frequency of the waves that experience the maximum reflection is shifted by the current, as the surface wavenumber $k$ changes for a fixed absolute frequency. Over sandy continental shelves, tidal currents are known to generate sandwaves with scales comparable to those of surface waves, with bottom elevation spectra that roll-off sharply at high wavenumbers. Application of the theory to such a real topography suggests that scattering mainly results in a broadening of the directional wave spectrum, i.e. forward scattering, while back-scattering is generally weaker. The current may strongly influence surface gravity wave scattering by selecting different bottom scales, with widely different spectral densities due the sharp bottom spectrum roll-off.

\section{Introduction}

Following the early observations of Heathershaw (1982), a considerable body of knowledge has been accumulated on the scattering of small amplitude surface gravity waves by periodic bottom topography. An asymptotic theory for small bottom amplitudes, that 
reproduces the observed scattering of monochromatic waves over a few sinusoidal bars, was put forward by Mei (1985), leading to practical phase-resolving equations that may be used to model this phenomenon for more general bottom shapes (Kirby 1986). For sinusoidal bottoms of wavenumber $l$, Mei (1985) proposed an approximate analytical solution. In two dimensions (one horizontal and the vertical) this solution yields simple expressions for the wave amplitude reflection coefficient $R$, as a function of the mismatch between the wavenumber of the surface waves $k$ and the resonant value $l / 2$, for which $R$ is maximum due to Bragg resonance. Beyond a cut-off value of that mismatch, it was found that the incident and reflected wave amplitudes oscillate in space instead of decreasing monotonically from the incident region. In three dimensions the Bragg resonance condition becomes $\boldsymbol{k}=\boldsymbol{l}+\boldsymbol{k}^{\prime}$ and $\omega=\omega^{\prime}$, with $\omega$ and $\omega^{\prime}$ the wave radian frequencies corresponding to the wavenumber vectors $\boldsymbol{k}$ and $\boldsymbol{k}^{\prime}$ through the linear dispersion relation.

Other contributions have shown that higher-order theories are necessary to represent the sub-harmonic resonance observed over a bottom that is a superposition of two components of different wavelengths (Guazzelli, Rey \& Belzons 1992). Such sub-harmonic resonance was found to have as large an effect as the lowest order resonance for bottom amplitudes of only $25 \%$ of the water depth, due to a general stronger reflection for relatively longer waves. However, these amplitude evolution equations are still prohibitively expensive for investigating the propagation of random waves over distances larger than about 100 wavelengths, and the details of the bottom are typically not available over large areas. Besides, a consistent phase-averaged wave action evolution equation is also necessary for the investigation of the long waves associated with short wave groups (Hara \& Mei 1987).

The large scale behaviour of the wave field may rather be represented by the evolution of the wave action spectrum assuming random phases. Such an approach was already proposed by Hasselmann (1966) and Elter \& Molyneux (1972) for the calculation of windwave and tsunami propagation. A proper theory for the evolution of the wave spectrum can be obtained from a solvability condition, a method similar to that of Mei (1985) and Kirby (1988), but applied to the action spectral densities instead of the amplitudes of monochromatic waves. In the absence of currents the correct form of that equation was first obtained by Ardhuin \& Herbers (2002, hereinafter referred to as AH) using a two scale approach. They decomposed the water depth $H-h$ in a slowly varying depth $H$, that causes shoaling and refraction, and a rapidly varying perturbation $h$ with zero mean, that causes scattering. This equation is formally similar to general transport equations for waves in random media (e.g. Ryzhik, Papanicolaou \& Keller 1996), although the waves considered here propagate only in the two horizontal dimensions. The resulting scattering was shown to be consistent with the dramatic increase of the directional width of the wave spectra observed on the North Carolina continental shelf (Ardhuin et al. 2003a, 2003b).

Recently, Magne et al. (2005, hereinafter referred to as MAHR) showed that AH's theory gives the same damping of incident waves as the Green function solution of Pihl, Mei \& Hancock (2002), applied to any two dimensional topography, random or not. Investigating the applicability limits of the scattering term of AH, MAHR also performed numerical calculations, comparing AH's theory to the accurate matched-boundary model of Rey (1992) that uses a decomposition of the bottom in a series of steps, including evanescent modes. The numerical results show that AH's theory is generally limited by the relative bottom amplitude $\eta=\max (h) / H$ rather than the bottom slope. In particular, AH's theory predicts accurate reflections, with a relative error of order $\eta$, even for isolated steps that have an infinite slope (MAHR).

The resulting expression of the Bragg scattering term is consistent with results for scat- 
tering of acoustic and electromagnetic waves obtained by the small perturbation method, valid in the limit of small $k \max (h)$ where $k$ is the wavenumber of the propagating waves (Rayleigh 1896, see Elfouhaily \& Guerin 2004 for a review of this and other approximations). Since there is no scattering for $k H \gg 1$, as the waves do not 'feel' the bottom, the small parameter $\eta=\max (h) / H$ may be used in the context of surface gravity waves, instead of the more general $k \max (h)$. For $\eta \ll 1$, the scattering strength is thus entirely determined by the bottom elevation variance spectrum at the bottom scales resonant with the incident waves.

Based on these results, Mei's (1985) theory should yield the same reflection coefficient as AH's theory in the limit of small bottom amplitudes. Yet, AH predict that the wave amplitude in 2D would decay monotonically, which is not compatible with the oscillatory nature of Mei's theory for large detunings from resonance. Further, outside of the surf zone and the associated multiple bar systems, the application of AH's theory is most relevant in areas where the bottom topography changes significantly on the scale of the wavelengths of swells. This often corresponds, over sand, to the presence of sandwaves. These sandwaves are generated by currents, and particularly by tidal currents (e.g. Dalrymple Knight \& Lambiase 1978; Idier, Erhold \& Garlan 2002). It is thus logical to include the effects of currents in any theory for wave scattering over a random bottom. Kirby (1988) developed such a theory for monochromatic waves over a sinusoidal bottom and a slowly varying mean current, extending Mei's (1985) work. The geometry of the resonant wavenumbers is modified in that case, with with incident and reflected waves having the same absolute frequency, but different wavenumber magnitudes if incident and reflected waves propagate at different angles relative to the current direction. Kirby (1988) also considered the short scale fluctuations of the current, due to the sinusoidal bottom, that may be interpreted as a separate scattering mechanism, and generalized further to any irrotational current fluctuations, leading to results similar to those obtained for gravity-capillary waves by Bal \& Chou (2002).

The present paper thus deals with these two questions. An extension of AH's theory for surface gravity wave scattering in the presence of irrotational currents with uniform mean velocities is provided in $\S 2$, and the differences between this theory and those of Mei (1985) and Kirby (1988) are discussed in detail in $\S 3$. Expected oceanographic effects of scattering in the presence of a current are investigated in $\S 4$, using a spectral phase-averaged numerical model, predicting the evolution of the wave action spectrum, and detailed measurements of the topography in the southern North Sea. Conclusions follow in $\S 5$.

\section{Theory}

\subsection{General formulation}

The variation in the action spectral density due to wave-bottom scattering is derived following the method of $\mathrm{AH}$, now including the effect of a uniform mean current. The method is identical to that of Kirby (1988) with the difference that an equation for the spectral wave action is sought instead of one for the wave amplitudes. Thus intermediate results are identical to those of Kirby (1988). Since the wave action is a quadratic function in the wave amplitude, we will naturally consider the wave potential up to second order in the normalized bottom amplitude $\eta$, in order to have all wave action terms to order $\eta^{2}$. The only important terms in this type of calculation are the 'secular terms', i.e. the harmonic oscillator solutions for the wave potential forced at resonance, with an amplitude that grows unbounded in time. We shall thus obtain a rate of change of the 


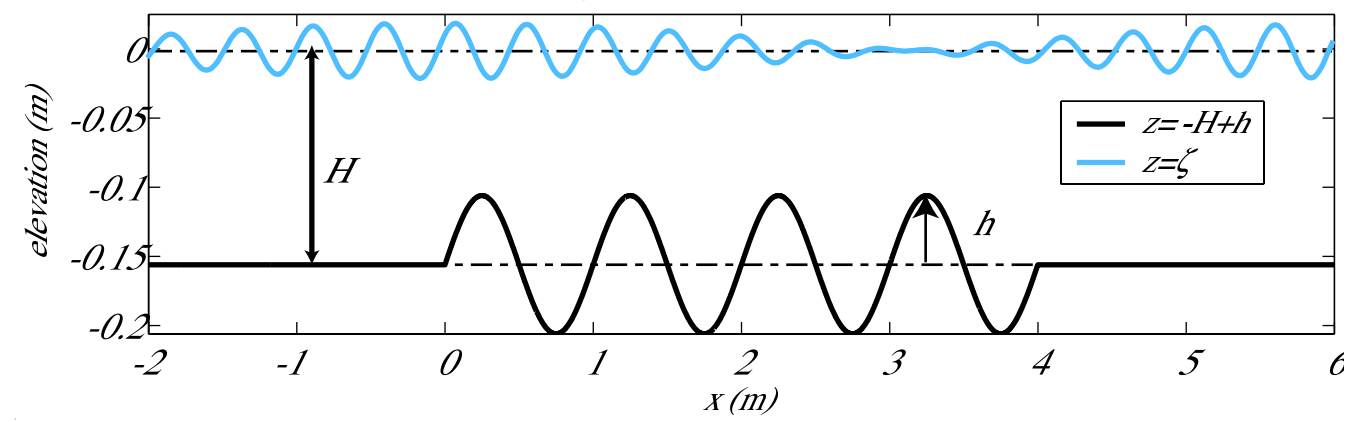

FiguRE 1. Definition sketch of the mean water depth $H$, and relative bottom elevation $h$, for one particular case of a sinusoidal bottom investigated in $\S 3$.

action from the equality of all the secular terms. The particularity of the random wave approach is also that we will consider all possible couplings between wave components, and not just two wave trains. With random waves, secularity is limited to a sub-space of the wavenumber plane that generally has a zero measure. Thus the near-resonant terms, once integrated across the resonant singularity, are the ones that provide the secular terms for random waves. This integration assumes that the spectral properties are continuous, a real theoretical problem for nonlinear wave-wave interactions (e.g. Benney \& Saffmann 1966, Onorato et al. 2004). Here we shall see that the only relevant condition is that the bottom spectrum be continuous, at least in one dimension. This is obviously satisfied by any real topography, since a truly infinite sinusoidal bottom of wavelength $L$, with an infinite spectral density at the wavenumber $2 \pi / L$, is not to be found, even in the laboratory.

We consider weakly nonlinear random waves propagating over an irregular bottom with a constant mean depth $H$ and mean current $\mathbf{U}$, and random topography $h(\boldsymbol{x})$, with $\boldsymbol{x}$ the horizontal position vector, so that the bottom elevation is given by $z=-H+h(\boldsymbol{x})$ where $z$ is the elevation relative to the still water level. The bottom undulations cause a stationary random small-scale current fluctuation $(\mathbf{u}(\boldsymbol{x}, z), w(\boldsymbol{x}, z))$ deriving from a potential $\phi_{c}$. The free surface is at $z=\zeta(\boldsymbol{x}, t)=\zeta(\boldsymbol{x}, t)$. Extension to mean current and mean depth variations on a large scale follows from a standard two-scale approximation, identical to that of by Kirby (1988). This is not included in the present derivation for the sake of simplicity.

The maximum surface slope is characterized by $\varepsilon$ and we shall assume that $\varepsilon^{3} \ll \eta^{2}$ so that the bottom scattering contributions to the wave action to order $\eta^{2}$ are much larger than the resonant non-linear four wave interactions (Hasselmann 1962) that shall be neglected. Such interactions could also be allowed in the present calculation providing an additional source of scattering with the known form due to cubic non-linearities. For shallow water waves $(k H<<1)$ a stricter inequality is needed to prevent triad wavewave interactions to enter the action evolution equation at the same order as bottom scattering.

The solution is obtained in a frame of reference moving with the mean current vector $\boldsymbol{U}$, which has the advantage of removing the convective terms due to the mean current velocity. The corresponding transformation of the horizontal coordinates is $\boldsymbol{x}^{\prime}=\boldsymbol{x}+\boldsymbol{U} t$, where $\boldsymbol{x}$ and $\boldsymbol{x}^{\prime}$ are the coordinates in the moving and fixed frames, respectively. As a result of this transformation, the bottom is moving, and the bottom boundary condition for the velocity potential is modified. The governing equations consist of Laplace's equation for the velocity potential, which includes both wave and current motions, the bottom 
kinematic boundary conditions, and Bernoulli's equation with the free surface kinematic boundary condition. Assuming that the atmospheric pressure is zero for simplicity, and neglecting surface tension, one has

$$
\begin{aligned}
\nabla^{2} \phi+\frac{\partial^{2} \phi}{\partial z^{2}} & =0 \text { for }-H+h \leqslant z \leqslant \zeta \\
\frac{\partial \phi}{\partial z} & =\frac{\partial h}{\partial t}+\nabla \phi \cdot \nabla h \quad \text { at } z=-H+h \\
\frac{\partial \phi}{\partial t}+g \zeta & =-\frac{1}{2}|\nabla \phi|^{2}-\frac{1}{2}\left(\frac{\partial \phi}{\partial z}\right)^{2}+c(t) \text { at } z=\zeta \\
\frac{\partial \phi}{\partial z} & =\frac{\partial \zeta}{\partial t}+\nabla \phi \cdot \nabla \zeta \text { at } z=\zeta
\end{aligned}
$$

with $c(t)$ a function of time only, to be determined. The symbol $\boldsymbol{\nabla}$ represents the usual gradient operator restricted to the two horizontal dimensions. The latter two equations may be combined to remove the linear part in $\zeta$. Taking $\partial(2.3) / \partial t+g(2.4)$, yields,

$$
\begin{array}{r}
\frac{\partial^{2} \phi}{\partial t^{2}}+g \frac{\partial \phi}{\partial z}=g \boldsymbol{\nabla} \phi \cdot \nabla \zeta-\frac{\partial \zeta}{\partial t} \frac{\partial^{2} \phi}{\partial z \partial t}-\left(1+\frac{\partial \zeta}{\partial t} \frac{\partial}{\partial z}\right)\left[\nabla \phi \cdot \frac{\partial \boldsymbol{\nabla} \phi}{\partial t}+\frac{\partial \phi}{\partial z} \frac{\partial^{2} \phi}{\partial t \partial z}\right] \\
\text { at } z=\zeta \cdot(2.5)
\end{array}
$$

Following Hasselmann (1962), we approximate $h$ and $\phi$ with discrete sums over Fourier components, and take the limit to continuous integrals after deriving expressions for the evolution of the phase-averaged wave action. We look for a velocity potential solution in the form

$$
\phi(\boldsymbol{x}, z, t)=\sum_{\boldsymbol{k}, s} \widehat{\Phi}_{\boldsymbol{k}}^{s}(z, \gamma t) \mathrm{e}^{\mathrm{i}[\boldsymbol{k} \cdot \boldsymbol{x}-s \sigma t]}=\sum_{\boldsymbol{k}, s} \Phi_{\boldsymbol{k}}^{s}(t) \frac{\cosh [k(z+H)]}{\cosh (k H)} \mathrm{e}^{\mathrm{i} \boldsymbol{k} \cdot \boldsymbol{x}}+\ldots,
$$

where $\sigma$ is the radian frequencies in the moving frame, $\boldsymbol{k}$ is the surface wavenumber, with magnitude $k$, and $s$ is a sign index equal to 1 or -1 . In the moving frame of reference, $s=1$ for wave components that propagate in the direction of the vector $\boldsymbol{k}$, and $s=-1$ for components that propagate in the opposite direction. Thus the radian frequency in the fixed frame is $\omega=\sigma+s \boldsymbol{k} \cdot \boldsymbol{U}$. The amplitudes $\widehat{\Phi}_{\boldsymbol{k}}^{s}$ are slowly modulated in time, with a slowness defined by the small parameter $\gamma$. Because $\phi$ is a real quantity we also have $\overline{\widehat{\Phi}_{\boldsymbol{k}}^{s}}=\widehat{\Phi}_{-\boldsymbol{k}}^{-s}$, where the overbar denotes the complex conjugate. Thus the double decomposition made in (2.6) in wavenumber $\boldsymbol{k}$ and propagation direction + or - replaces a more general decomposition in wavenumber and frequency that would be necessary if nonlinear dispersive effects were included. Here the frequency $\sigma$ is always related to $k$ via the linear dispersion relation.

In the alternative decomposition with amplitudes $\Phi_{k}^{s}$ that contain the fast time variation, only the part of the solution that has the vertical structure of Airy waves has been given explicitly. The other part, represented by '...', will be found to be negligible for small bottom amplitudes. Our rather archaic use of the $s$ index to distinguish the wave propagation direction is preferred here to the more modern use of the Hamiltonian variables that combine elevation and potential at the free surface, widely used for wave-wave interaction studies (e.g. Janssen 2004). The complexity of the Hamiltonian variables appears unnecessary for the linear waves considered here.

Expanding the bottom boundary condition and wave potential in powers of $\eta=$ $\max (h) / H$,

$$
\phi=\phi_{0}+\phi_{1}+\phi_{2}+\ldots
$$


where each term $\phi_{i}$ is of order $\eta^{i}$. The boundary conditions (2.5) and (2.2) are expressed at $z=0$ and $z=-H$, respectively, using Taylor series of $\phi$ about $z=-H$ and $z=0$.

Unless stated otherwise, these potential amplitudes will be random variables. Since we are solving for $\phi$ seeking an equation the for wave action $N$, we must relate $N$ to $\phi$. Accurate to second order in $\varepsilon$ and $\eta$ (see Andrews \& McIntyre 1978 for the general expression of $N$ ) we have $N=E / \sigma$ for a monochromatic wave of surface elevation variance $E$ and intrinsic frequency $\sigma$, in which, following the common usage in nonaccelerated reference frames, the gravity $g$ is left out, so that the action has units of meters squared times second. For general waves, the variance $E$ may be written as

$$
E(t)=\left\langle\left(\zeta_{0}+\zeta_{1}+\zeta_{2}+\ldots\right)^{2}\right\rangle=\left\langle\zeta_{0}^{2}+2 \zeta_{0} \zeta_{1}+\left(\zeta_{1}^{2}+2 \zeta_{0} \zeta_{2}\right)+\ldots\right\rangle,
$$

where $\langle\cdot\rangle$ denotes an average over flow realizations, and $\zeta_{i}$ is the surface elevation solution of order $\eta^{i}$, and terms of like order in $\eta$ have been grouped. Each of these terms may be expanded in this form

$$
\left\langle\zeta_{0}^{2}\right\rangle=\sum_{\boldsymbol{k}, s}\left|Z_{0, \boldsymbol{k}}^{s}\right|^{2}=2 \sum_{\boldsymbol{k}} Z_{0, \boldsymbol{k}}^{+} Z_{0,-\boldsymbol{k}}^{-}
$$

For free wave components, the elevation amplitude is proportional to the velocity potential amplitude

$$
Z_{i, k}^{s}=\mathrm{i} s \sigma \Phi_{j, k}^{s} / g
$$

so that the elevation co-variances are proportional to the co-variances $F_{i, j, k}^{\Phi}$ of the surface velocity potential,

$$
F_{i, j, k}^{\Phi}=\left\langle\Phi_{i, k}^{+} \Phi_{j,-\boldsymbol{k}}^{-}+\Phi_{i,-\boldsymbol{k}}^{-} \Phi_{j, \boldsymbol{k}}^{+}\right\rangle
$$

The contribution of the complex conjugate pairs of components $(\boldsymbol{k},+)$ and $(-\boldsymbol{k},-)$ are combined in (2.11) so that the covariance $F_{i, j, k}^{\Phi}$ correspond to that of all waves with wavenumber magnitude $k$ propagating in the direction of $\boldsymbol{k}$. In the limit of small wavenumber separation, a continuous slowly-varying cross-spectrum can be defined (e.g. Priestley 1981, ch.11; see also AH),

$$
F_{i, j}^{\Phi}(\boldsymbol{k})=\lim _{|\Delta k| \rightarrow 0} \frac{F_{i, j, k}^{\Phi}}{\Delta k_{x} \Delta k_{y}} .
$$

The definition of all spectral densities are chosen so that the integral over the entire wavenumber plane yields the total covariance of $\phi_{i}$ and $\phi_{j}$.

Finally, $N_{i, j}(\boldsymbol{k})$ is defined as the $(i+j)^{\text {th }}$ order depth-integrated wave action contribution from correlation between $i^{\text {th }}$ and $j^{\text {th }}$ order components with wavenumber $k$. From (2.8) and (2.10) one has,

$$
N_{i, j}(\boldsymbol{k})=\frac{k}{g \sigma} F_{i, j}^{\Phi}(\boldsymbol{k}) \tanh (k H) .
$$

The spectral wave action is thus,

$$
N(\boldsymbol{k})=\sum_{i=0}^{\infty} N_{i}(\boldsymbol{k})=\sum_{i=0}^{\infty} \sum_{j=0}^{i} N_{i, i-j}(\boldsymbol{k}) .
$$

Defining $G_{l}$ as the amplitude of the Fourier component of wavenumber $\boldsymbol{l}$, the bottom elevation is given by

$$
h(\boldsymbol{x})=\sum_{\boldsymbol{l}} G_{\boldsymbol{l}} \mathrm{e}^{\mathrm{i} \boldsymbol{l} \cdot[\boldsymbol{x}+\boldsymbol{U} t]},
$$


with a summation on the entire wavenumber plane. Because $h$ is real, $\overline{G_{-l}}=G_{\boldsymbol{l}}$. The bottom elevation spectrum in discrete form is given by $F_{\boldsymbol{l}}^{G}=\left\langle G_{\boldsymbol{l}} G_{-\boldsymbol{l}}\right\rangle$ and in continuous form by

and verifies,

$$
F^{B}(\boldsymbol{l})=\lim _{|\Delta l| \rightarrow 0} \frac{F_{l}^{G}}{\Delta l_{x} \Delta l_{y}}
$$

$$
\int_{-\infty}^{\infty} \int_{-\infty}^{\infty} F^{B}(\boldsymbol{l}) \mathrm{d} l_{x} \mathrm{~d} l_{y}=\lim _{L \rightarrow \infty} \frac{1}{L^{2}} \int_{-L / 2}^{L / 2} \int_{-L / 2}^{L / 2} h^{2}(x, y) \mathrm{d} x \mathrm{~d} y
$$

Now that the scene is set, we shall solve for the velocity potential $\phi$ in the frame of reference moving with the mean current, and use (2.13) to estimate the action spectra density at each successive order. In the course of this calculation, $\phi$ will appear as the sum of many terms, some of which are secular (these are the 'resonant terms' in Hasselmann's terminology), i.e. with growing amplitudes in time. Most importantly among these are those that lead to resonant terms in $N$. All other terms are bounded in time and thus do not contribute to the long-term evolution of the wave spectrum, i.e. on the scale of several wave periods, and shall be neglected (see Hasselmann 1962).

\subsection{Zeroth-order solution}

In the moving frame of reference, the governing equations for $\phi_{0}$ are identical to those in the fixed frame in the absence of current. The solution is thus

$$
\phi_{0}=\sum_{\boldsymbol{k}, s} \frac{\cosh (k(z+H))}{\cosh (k H)} \Phi_{0, \boldsymbol{k}}^{s} \mathrm{e}^{\mathrm{i}[\boldsymbol{k} \cdot \boldsymbol{x}-s \sigma t]},
$$

where the intrinsic frequency $\sigma$ is the positive root of the linear dispersion relation,

$$
\sigma^{2}=g k \tanh (k H) \text {. }
$$

\subsection{First-order solution}

Surface non-linearity becomes relevant at first order due to a coupling between the zeroth order solution and current-induced first order terms. Including all powers of $\eta$, the expansion of the surface boundary condition to order $\varepsilon^{2}$ gives, at $z=0$,

$$
\begin{aligned}
\frac{\partial \phi}{\partial t^{2}}+g \frac{\partial \phi}{\partial z}= & -\zeta \frac{\partial^{3} \phi}{\partial t^{2} \partial z}-g \zeta \frac{\partial^{2} \phi}{\partial z^{2}}-\frac{\partial \zeta}{\partial t} \frac{\partial^{2} \phi}{\partial z \partial t}+\nabla \phi \cdot\left(g \nabla \zeta-\frac{\partial \boldsymbol{\nabla} \phi}{\partial t}\right)-\frac{\partial \phi}{\partial z} \frac{\partial^{2} \phi}{\partial t \partial z} \\
& +c_{1}^{\prime}(t)+O\left(\varepsilon^{3}\right)
\end{aligned}
$$

The equations at order $\eta$ are

$$
\begin{aligned}
\nabla^{2} \phi_{1}+\frac{\partial^{2} \phi_{1}}{\partial z^{2}} & =0 \text { for } \quad-H \leqslant z \leqslant 0, \\
\frac{\partial \phi_{1}}{\partial z} & =-h \frac{\partial^{2} \phi_{0}}{\partial z^{2}}+\nabla \phi_{0} \cdot \nabla h+\frac{\partial h}{\partial t} \quad \text { at } \quad z=-H,
\end{aligned}
$$

and, at $z=0$, expansion of $(2.20)$ to first order in $\eta$ yields,

$$
\begin{aligned}
\frac{\partial^{2} \phi_{1}}{\partial t^{2}}+g \frac{\partial \phi_{1}}{\partial z}= & \overbrace{g\left(\boldsymbol{\nabla} \phi_{0} \cdot \nabla \zeta_{1}-\zeta_{1} \frac{\partial^{2} \phi_{0}}{\partial z^{2}}\right)}^{\mathrm{I}}+\overbrace{g\left(\boldsymbol{\nabla} \phi_{1} \cdot \boldsymbol{\nabla} \zeta_{0}-\zeta_{0} \frac{\partial^{2} \phi_{1}}{\partial z^{2}}\right)}^{\text {II }}-\overbrace{\mathrm{VI} \phi_{1} \cdot \frac{\partial \boldsymbol{\nabla} \phi_{0}}{\partial t}}^{\text {III }} \\
& -\nabla \phi_{0} \cdot \frac{\partial \boldsymbol{\nabla} \phi_{1}}{\partial t}-\left(\frac{\partial \phi_{1}}{\partial z}+\frac{\partial \zeta_{1}}{\partial t}\right) \frac{\partial^{2} \phi_{0}}{\partial t \partial z}-2 \frac{\partial \phi_{0}}{\partial z} \frac{\partial^{2} \phi_{1}}{\partial t \partial z}-\zeta_{1} \frac{\partial^{3} \phi_{0}}{\partial t^{2} \partial z}-\zeta_{0} \frac{\partial^{3} \phi_{1}}{\partial t^{2} \partial z}+N L_{1}
\end{aligned}
$$


where the terms $N L_{1}$, not written explicitly (see Hasselmann 1962 eq. 1.11-1.12), are quadratic products of the zeroth-order solution. Since no gravity waves satisfy both $\sigma=\sigma_{1} \pm \sigma_{2}$ and $\boldsymbol{k}=\boldsymbol{k}_{1} \pm \boldsymbol{k}_{2}, N L_{1}$ forces a non-resonant wave solution $\phi_{1}^{\text {nl }}$ that will be neglected because it does not modify our second order wave action balance, thanks to the choice $\varepsilon<\eta$. The spatially uniform term $c_{1}^{\prime}(t)$ has been incorporated into $N L_{1}$ and is also of second order in the wave slope, and does not lead to resonances. That term, omitted by Hasselmann (1962), is responsible for generating microseisms (e.g. Longuet-Higgins 1950).

The first-order system of equations is non-linear due to the surface boundary condition (2.23). However, all the right hand side terms of (2.23) are of order $\varepsilon \eta \phi_{0}$, and thus negligible, provided that $\phi_{1}$ is of order $\eta \phi_{0}$. Without $\partial h / \partial t$ in (2.22) this would be the case, since the other forcing terms are all proportional to $\eta \phi_{0}$. However, as suggested by anonymous reviewers, $\partial h / \partial t$ introduces an external forcing. We thus first give the solution $\left(\phi_{1 c}, \zeta_{1 c}\right)$ forced by $\partial h / \partial t$ only, in the right hand side of $(2.22)$. This solution is physically identical to mean current perturbation caused by the bottom topography and given by Kirby (1988, his eq. 2.9) for a sinusoidal bottom. With a more general bottom, it is

$$
\phi_{1 c}=\mathrm{i} \sum_{\boldsymbol{l}} \boldsymbol{U} \cdot \boldsymbol{l} \frac{G_{\boldsymbol{l}}}{l \alpha_{\boldsymbol{l}}}\left\{\beta_{\boldsymbol{l}} \cosh [l(z+H)]+\alpha_{\boldsymbol{l}} \sinh [l(z+H)]\right\} \mathrm{e}^{\mathrm{i} \boldsymbol{l} \cdot(\boldsymbol{x}+\boldsymbol{U} t)},
$$

where

and

$$
\alpha_{\boldsymbol{l}}=\frac{(\boldsymbol{U} \cdot \boldsymbol{l})^{2}}{g l}-\tanh (l h)
$$

$$
\beta_{\boldsymbol{l}}=1-\tanh (l h) \frac{(\boldsymbol{U} \cdot \boldsymbol{l})^{2}}{g l} .
$$

The corresponding surface elevation oscillations, given by (2.3), are second order in the Froude number $\mathrm{Fr}=U /(g h)^{1 / 2}$, and $180^{\circ}$ out of phase with the bottom oscillations for slow currents when $\alpha<0$ (Kirby 1988, eq. 2.10),

$$
\zeta_{1 c}=\sum_{\boldsymbol{l}} \frac{(\boldsymbol{U} \cdot \boldsymbol{l})^{2} G_{\boldsymbol{l}}}{g \alpha_{\boldsymbol{l}} \cosh (l h)} \mathrm{e}^{\mathrm{i} l \cdot(\boldsymbol{x}+\boldsymbol{U} t)}
$$

From (2.24), the following expression are derived,

$$
\begin{gathered}
\phi_{1 c}(z=0)=\mathrm{i} \sum_{\boldsymbol{l}} \boldsymbol{U} \cdot \boldsymbol{l} \frac{G_{\boldsymbol{l}}}{l \alpha_{\boldsymbol{l}} \cosh (l h)} \mathrm{e}^{\mathrm{i} \boldsymbol{l} \cdot(\boldsymbol{x}+\boldsymbol{U} t)}, \\
\frac{\partial \phi_{1 c}}{\partial z}(z=0)=\frac{\partial \zeta_{1 c}}{\partial t}=\mathrm{i} \sum_{\boldsymbol{l}}(\boldsymbol{U} \cdot \boldsymbol{l})^{3} \frac{G_{\boldsymbol{l}}}{g \alpha_{\boldsymbol{l}} \cosh (l h)} \mathrm{e}^{\mathrm{i} \boldsymbol{l} \cdot(\boldsymbol{x}+\boldsymbol{U} t)} .
\end{gathered}
$$

These shall be particularly useful for plugging into (2.23).

We can now obtain the general solution to our equations (2.21) -2.23 ) by the following superposition of the previous solution with free and bound (i.e. non-resonant) wave components, with amplitudes $\Phi_{1, \boldsymbol{k}}^{s}$ and $\Phi_{1, \boldsymbol{k}}^{\mathrm{si}, s}$ respectively,

$$
\phi_{1}=\phi_{1 c}+\sum_{\boldsymbol{k}, s}\left[\frac{\cosh [k(z+H)]}{\cosh (k H)} \Phi_{1, \boldsymbol{k}}^{s}(t)+\frac{\sinh [k(z+H)]}{\cosh (k H)} \Phi_{1, \boldsymbol{k}}^{\mathrm{si}, s}(t)\right] \mathrm{e}^{\mathrm{i} \boldsymbol{k} \cdot \boldsymbol{x}},
$$


where the last two terms corresponds to the solution to the forcing by all the right hand side terms except for $\partial h / \partial t$. Because $\phi_{1 c}$ and $\zeta_{1 c}$ are the only terms that may be larger than $\varepsilon \eta \phi_{0}$, all others are neglected in the right-hand side of (2.23).

Substitution of (2.30) in the bottom boundary condition (2.22) yields

$$
\frac{k}{\cosh (k H)} \Phi_{1, \boldsymbol{k}}^{\mathrm{si}, s}(t)=-\sum_{\boldsymbol{k}^{\prime}} \frac{\boldsymbol{k}^{\prime} \cdot \boldsymbol{k}}{\cosh \left(k^{\prime} H\right)} \Phi_{1, \boldsymbol{k}^{\prime}}^{s} G_{\boldsymbol{k}-\boldsymbol{k}^{\prime}} \mathrm{e}^{\mathrm{i}\left[\left(\boldsymbol{k}-\boldsymbol{k}^{\prime}\right) \cdot \boldsymbol{U}-s \sigma^{\prime}\right] t} .
$$

Replacing now (2.30) in the surface boundary condition 2.23), yields an equation for $\Phi_{1, \boldsymbol{k}}^{s}$. Using $\omega=\sigma+\boldsymbol{k} \cdot \boldsymbol{U}$ and $\omega^{\prime}=\sigma^{\prime}+\boldsymbol{k}^{\prime} \cdot \boldsymbol{U}$, it writes

$$
\left(\frac{d^{2}}{d t^{2}}+\sigma^{2}\right) \Phi_{1, \boldsymbol{k}}^{s}(t)=\sum_{\boldsymbol{k}^{\prime}} M^{s}\left(\boldsymbol{k}, \boldsymbol{k}^{\prime}\right) \Phi_{0, \boldsymbol{k}^{\prime}} G_{\boldsymbol{k}-\boldsymbol{k}^{\prime}} \mathrm{e}^{\mathrm{i}\left[\boldsymbol{k} \cdot \boldsymbol{U}-s \omega^{\prime}\right] t},
$$

with

$$
M^{s}\left(\boldsymbol{k}, \boldsymbol{k}^{\prime}\right)=\left\{g k-\left[\boldsymbol{k} \cdot \boldsymbol{U}-s \omega^{\prime}\right]^{2} \tanh (k H)\right\} \frac{\boldsymbol{k}^{\prime} \cdot \boldsymbol{k}}{k} \frac{\cosh (k H)}{\cosh \left(k^{\prime} H\right)}+M_{c 1}^{s}\left(\boldsymbol{k}, \boldsymbol{k}^{\prime}\right)
$$

where $M_{c 1}^{s}$ is given by all the right-hand side terms in 2.23) and thus corresponds to the scattering induced by current and current-induced surface elevation variations. Anticipating resonance, we only give the form of $M_{c 1}^{s}=M_{c}^{s}$ for $\sigma=\sigma^{\prime}-s \boldsymbol{l} \cdot \boldsymbol{U}$, with $\boldsymbol{l}=\boldsymbol{k}-\boldsymbol{k}^{\prime}$,

$$
M_{c}^{s}\left(\boldsymbol{k}, \boldsymbol{k}^{\prime}\right)=\frac{\left\{s g^{2} \boldsymbol{U} \cdot \boldsymbol{l}\left(\sigma^{\prime} \boldsymbol{l} \cdot \boldsymbol{k}+\sigma \boldsymbol{l}^{(\mathrm{b})} \boldsymbol{k}^{\prime}\right)-(\boldsymbol{U} \cdot \boldsymbol{l})^{2}[g^{2} \boldsymbol{k}^{(\mathrm{c})} \cdot \boldsymbol{k}^{\prime}-\overbrace{\sigma \sigma^{\prime}\left(\sigma \sigma^{\prime}+(\boldsymbol{U} \cdot \boldsymbol{l})^{2}\right.})\right]}{(\mathrm{d})},
$$

in which the term (a) is given by the term (II) in 2.20 ), (b) is given by (III) and (IV), (c) is given by (I), and (d) is given by (V)-(VIII). Because we are first solving the problem to order $\eta$, it is natural that our solution is a linear superposition of the solutions found by Kirby (1988) for a single bottom component. Indeed, $M_{c}\left(\boldsymbol{k}, \boldsymbol{k}^{\prime}\right)=-4 \omega \Omega_{c} / D$, with $\Omega_{c}$ the interaction coefficient of Kirby (1988, eq. $4.22 \mathrm{~b})$ and $D$ his bottom amplitude, here $G_{l}=\mathrm{i} D / 2$.

The solution to the forced harmonic oscillator equation (2.32) is

$$
\Phi_{1, \boldsymbol{k}}^{s}(t)=\sum_{\boldsymbol{k}^{\prime}} M^{s}\left(\boldsymbol{k}, \boldsymbol{k}^{\prime}\right) \Phi_{0, \boldsymbol{k}^{\prime}}^{s} G_{\boldsymbol{k}-\boldsymbol{k}^{\prime}} f_{1}\left(\sigma, \boldsymbol{l} \cdot \boldsymbol{U}-s \sigma^{\prime} ; t\right)
$$

where $\boldsymbol{l}=\boldsymbol{k}-\boldsymbol{k}^{\prime}$, and the function $f_{1}$ is defined in Appendix A.

\subsubsection{First order action}

The lowest order perturbation of the wave action by scattering involves the order $\eta$ covariances

$$
F_{1,0, \boldsymbol{k}}^{\Phi}+F_{0,1, \boldsymbol{k}}^{\Phi}=4 \operatorname{Re}\left(\left\langle\Phi_{0, \boldsymbol{k}}^{+} \Phi_{1,-\boldsymbol{k}}^{-}\right\rangle\right)
$$

with Re denoting the real part. Including only the secular terms, we get

$$
F_{1,0, \boldsymbol{k}}^{\Phi}+F_{0,1, \boldsymbol{k}}^{\Phi}=4 \operatorname{Re}\left[\sum_{\boldsymbol{k}^{\prime}} M^{+}\left(\boldsymbol{k}, \boldsymbol{k}^{\prime}\right)\left\langle\Phi_{0, \boldsymbol{k}^{\prime}}^{+} \Phi_{0,-\boldsymbol{k}}^{-} G_{\boldsymbol{k}-\boldsymbol{k}^{\prime}}\right\rangle f_{1}\left(\sigma, \boldsymbol{l} \cdot \boldsymbol{U}-\sigma^{\prime} ; t\right) \mathrm{e}^{\mathrm{i} \sigma t}\right] .
$$

Although this term was assumed to be zero in AH, it is not zero for sinusoidal bottoms with partially standing waves, and may become significant at resonance due to the function $f_{1}$. In uniform conditions, the time evolution of the wave field requires that the 
non-stationarity must come into play. Thus $\gamma \approx \eta$ and the non-stationary term is given by $\mathrm{AH}$ (their appendix D),

$$
\frac{\partial\left[N_{1,0}^{\mathrm{ns}}(\boldsymbol{k})+N_{0,1}^{\mathrm{ns}}(\boldsymbol{k})\right]}{\partial t}=-\frac{\partial N_{0}(\boldsymbol{k})}{\partial t} .
$$

In order to simplify the discussion, we shall briefly assume that there is no current and that the waves are unidirectional. In that case, $\boldsymbol{k}^{\prime}=-\boldsymbol{k}$ and $M\left(\boldsymbol{k}, \boldsymbol{k}^{\prime}\right)=-g k^{2} / \cosh ^{2}(k H)$. Replacing (2.37) in (2.13) and combining it with (2.38) yields the action balance

$$
\frac{\partial N_{0, \boldsymbol{k}}}{\partial t}=\frac{\partial}{\partial t}\left[\frac{k}{g \sigma} \tanh (k H)\left(F_{1,0, \boldsymbol{k}}^{\Phi}+F_{0,1, \boldsymbol{k}}^{\Phi}\right)\right]=\operatorname{Im}\left(\frac{-4 k^{2} \sigma}{2 g \cosh ^{2}(k H)}\left\langle\Phi_{0, \boldsymbol{k}}^{+} \Phi_{0, \boldsymbol{k}}^{-} G_{-2 k}\right\rangle\right),
$$

with Im denoting the imaginary part.

For directionally spread random waves, with a current, and a real bottom (e.g. random or consisting of a finite series of sinusoidal bars), the evaluation of (2.37) is not simple. First of all, resonant terms given by $f_{1}$ only occur for $\sigma^{\prime}=\sigma+s \boldsymbol{l} \cdot \boldsymbol{U}$, that is $\omega=\omega^{\prime}$. Using $N(\boldsymbol{k})=N_{0}(\boldsymbol{k})[1+O(\eta)]$ and taking the limit to continuous surface and bottom spectra yields

$$
\frac{\partial N(\boldsymbol{k})}{\partial t}=S_{1}(\boldsymbol{k})=\int_{0}^{2 \pi} \frac{4 \boldsymbol{k} \cdot \boldsymbol{k}^{\prime}}{2 g \cosh (k H) \cosh \left(k^{\prime} H\right)} \operatorname{Im}\left[Z\left(\boldsymbol{k}, \boldsymbol{k}^{\prime}\right)\right] \mathrm{d} k_{x}^{\prime} \mathrm{d} \theta^{\prime},
$$

with the mixed surface bottom bispectrum $Z$ defined by

$$
Z\left(\boldsymbol{k}, \boldsymbol{k}^{\prime}\right)=\lim _{\Delta \boldsymbol{k} \rightarrow \infty}\left\langle\frac{\Phi_{1, \boldsymbol{k}}^{+} \Phi_{1,-\boldsymbol{k}^{\prime}}^{-} G_{-k-k^{\prime}}}{\Delta \boldsymbol{k} \Delta \theta^{\prime}}\right\rangle
$$

with $\boldsymbol{k}=k(\cos \theta, \sin \theta)$ and $\boldsymbol{k}^{\prime}=k\left(\cos \theta^{\prime}, \sin \theta^{\prime}\right)$. Z is similar to a classical bispectrum (e.g. Herbers et al. 2003) with one surface wave amplitude replaced by a bottom amplitude, and a similar expression is found for a non-zero current. The action balance (2.40) is generally not closed, and requires a knowledge of the wave phases that are not available in a phase-averaged model. The same type of coupling, although due to the large scale topography, also occurs in the stochastic equations for non-linear wave evolution derived by Janssen, Herbers \& Battjes (2006).

The contribution of the mixed bispectrum will thus be evaluated below, in order to investigate in which cases it may be neglected or parameterized. It is expected that $S_{1}$ is generally negligible because MAHR have neglected $S_{1}$, and still found a good agreement of the second order action balance with exact numerical solutions for the wave amplitude reflection coefficient.

\subsubsection{Second order action}

From the expansion (2.14), the second order action is $N_{2}(\boldsymbol{k})=N_{1,1}(\boldsymbol{k})+N_{0,2}(\boldsymbol{k})+$ $N_{2,0}(\boldsymbol{k})$. The first term can be estimated from $\phi_{1}$, using the covariance of the velocity potential amplitudes (2.11),

$$
F_{1,1, \boldsymbol{k}}^{\Phi}=2\left\langle\Phi_{1, \boldsymbol{k}}^{+} \Phi_{1,-\boldsymbol{k}}^{-}\right\rangle .
$$

Using (2.35), 2.42) can be re-written as

$$
\frac{F_{1,1, \boldsymbol{k}}^{\Phi}}{\Delta \boldsymbol{k}}=2 \sum_{\boldsymbol{k}^{\prime}}\left|M^{+}\left(\boldsymbol{k}, \boldsymbol{k}^{\prime}\right)\right|^{2} \frac{\left\langle\left|\Phi_{0, \boldsymbol{k}^{\prime}}^{+}\right|^{2}\right\rangle}{\Delta \boldsymbol{k}^{\prime}} \frac{\left\langle\left|G_{\boldsymbol{k}-\boldsymbol{k}^{\prime}} G_{-\boldsymbol{k}+\boldsymbol{k}^{\prime}}\right|^{2}\right\rangle}{\Delta \boldsymbol{k}}\left|f_{1}\left(\sigma, \boldsymbol{l} \cdot \boldsymbol{U}-\sigma^{\prime} ; t\right)\right|^{2} \Delta \boldsymbol{k}^{\prime}
$$


Taking the limit of $(2.43)$ when $\Delta \boldsymbol{k} \rightarrow 0$,

$$
F_{1,1}^{\Phi}(t, \boldsymbol{k})=\int_{-\infty}^{\infty} \int_{-\infty}^{\infty}\left|M^{+}\left(\boldsymbol{k}, \boldsymbol{k}^{\prime}\right)\right|^{2} F_{1,1}^{\Phi}\left(\boldsymbol{k}^{\prime}\right) F^{B}\left(\boldsymbol{k}-\boldsymbol{k}^{\prime}\right)\left|f_{1}\left(\sigma, \boldsymbol{l} \cdot \boldsymbol{U}-\sigma^{\prime} ; t\right)\right|^{2} \mathrm{~d} k_{x}^{\prime} \mathrm{d} k_{y}^{\prime} .
$$

Due to the singularity in $f_{1}$, and assuming that the rest of the integrand can be approximated by an anlytical function in the neighbourhood of the singularity $\omega^{\prime}=\omega$, which requires both bottom and surface elevation spectra to be continuous, the integral can be evaluated by using

$$
\left\langle f_{1}\left(\sigma, \boldsymbol{l} \cdot \boldsymbol{U}-\sigma^{\prime} ; t\right) f_{1}\left(\sigma,-\boldsymbol{l} \cdot \boldsymbol{U}+\sigma^{\prime} ; t\right)\right\rangle=\frac{\pi t}{4 \sigma^{2}}\left[\delta\left(\sigma^{\prime}-(\sigma+\boldsymbol{l} \cdot \boldsymbol{U})\right)+O(1)\right]
$$

$\delta$ is the one-dimension Dirac distribution, infinite where the argument is zero, and such that $\int \delta(x) A(x) \mathrm{d} x=A(0)$ for any continuous function $A$. In order to remove that singularity, the argument of $\delta$ maye be re-written as $\omega^{\prime}-\omega$, making explicit all the dependencies on $k^{\prime}$. Evaluation of the $\delta$ function is then performed by changing integration variables $\left(k_{x}^{\prime}, k_{y}^{\prime}\right)$ are changed to $\left(\omega^{\prime}, \theta^{\prime}\right)$, with a Jacobian $k^{\prime} \partial k^{\prime} / \partial \omega^{\prime}=k^{\prime 2} /\left(k^{\prime} C_{g}^{\prime}+\boldsymbol{k}^{\prime} \cdot \boldsymbol{U}\right)$. We thus have

$$
F_{1,1}^{\Phi}(t, \boldsymbol{k})=\frac{\pi t}{2 \sigma^{2}} \int_{0}^{2 \pi} \int_{\omega^{\prime}}\left|M^{+}\left(\boldsymbol{k}, \boldsymbol{k}^{\prime}\right)\right|^{2} F_{1,1}^{\Phi}\left(\boldsymbol{k}^{\prime}\right) \frac{k^{\prime} F^{B}\left(\boldsymbol{k}-\boldsymbol{k}^{\prime}\right)}{C_{g}^{\prime}+\boldsymbol{k}^{\prime} \cdot \boldsymbol{U}} \delta\left(\omega^{\prime}-\omega\right) d \omega^{\prime} \mathrm{d} \theta^{\prime}+O(1) .
$$

When $\omega=\omega^{\prime}$, the integrand simplifies. $M^{s}\left(\boldsymbol{k}, \boldsymbol{k}^{\prime}\right)$ is equal to $M\left(\boldsymbol{k}, \boldsymbol{k}^{\prime}\right)$, defined by

$$
M\left(\boldsymbol{k}, \boldsymbol{k}^{\prime}\right)=\frac{g \boldsymbol{k} \cdot \boldsymbol{k}^{\prime}}{\cosh (k H) \cosh \left(k^{\prime} H\right)}+M_{c}\left(\boldsymbol{k}, \boldsymbol{k}^{\prime}\right) \equiv M_{b}\left(\boldsymbol{k}, \boldsymbol{k}^{\prime}\right)+M_{c}\left(\boldsymbol{k}, \boldsymbol{k}^{\prime}\right),
$$

with $M_{c}=M_{c}^{+}$given by (2.34). Using the (2.13) relation between velocity potential and action, and evaluating the integral over $\omega^{\prime}$, one obtains

$$
N_{1,1}(t, \boldsymbol{k})=\frac{\pi t}{2} \int_{0}^{2 \pi} M^{2}\left(\boldsymbol{k}, \boldsymbol{k}^{\prime}\right) \frac{N_{0,0}\left(\boldsymbol{k}^{\prime}\right)}{\sigma \sigma^{\prime}} F^{B}\left(\boldsymbol{k}-\boldsymbol{k}^{\prime}\right) \frac{k^{\prime 2}}{k^{\prime} C_{g}^{\prime}+\boldsymbol{k}^{\prime} \cdot \boldsymbol{U}} d \theta^{\prime}+O(1) .
$$

Again we note the correspondance with the theory of Kirby (1988, eq. 4.21). Specifically, one has $M\left(\boldsymbol{k}, \boldsymbol{k}^{\prime}\right)=-4 \omega \Omega_{c} / D$, with $\Omega_{c}$ being Kirby's interaction coefficient.

\subsection{Second order potential and corresponding terms in $N_{2}$}

In order to estimate the other two terms that contribute to $N_{2}$, the second order potential $\phi_{2}$ must be obtained. It is a solution of

$$
\begin{gathered}
\nabla^{2} \phi_{2}+\frac{\partial^{2} \phi_{2}}{\partial z^{2}}=0 \quad \text { for } \quad-H \leqslant z \leqslant 0, \\
\frac{\partial \phi_{2}}{\partial z}=-h \frac{\partial^{2} \phi_{1}}{\partial z^{2}}-\frac{h^{2}}{2} \frac{\partial^{3} \phi_{0}}{\partial z^{3}}+\nabla \phi_{1} \cdot \nabla h+\nabla\left(h \frac{\partial \phi_{0}}{\partial z}\right) \cdot \nabla h \quad \text { at } \quad z=-H,
\end{gathered}
$$

that simplifies because odd vertical derivatives of $\phi_{0}$ are zero at $z=-H$,

$$
\frac{\partial \phi_{2}}{\partial z}=-h \frac{\partial^{2} \phi_{1}}{\partial z^{2}}+\nabla \phi_{1} \cdot \nabla h \quad \text { at } \quad z=-H
$$

and

$$
\frac{\partial^{2} \phi_{2}}{\partial t^{2}}+g \frac{\partial \phi_{2}}{\partial z}=\mathrm{i} \sum_{\boldsymbol{k}, s} 2 s \sigma \frac{\partial \Phi_{0, \boldsymbol{k}}^{s}}{\partial t} \mathrm{e}^{\mathrm{i}(\boldsymbol{k} \cdot \boldsymbol{x}-s \omega t)}+\mathrm{I}-\mathrm{VIII}+N L_{2} \quad \text { at } \quad z=0 .
$$


The terms I-VIII are identical to those in (2.23) with $\phi_{0}, \zeta_{0}, \phi_{1}, \zeta_{1}$ replaced by $\left(\phi_{1}-\phi_{1 c}\right)$, $\left(\zeta_{1}-\zeta_{1 c}\right), \phi_{2}$ and $\zeta_{2}$, respectively. All other non-linear terms have been grouped in $N L_{2}$. In order to yield contributions to the second order action $N_{2,0}$, terms must correlate with $\phi_{0}$ to give second-order terms in $\eta$ with non-zero means. For zeroth order components with random phases, inspection shows that $N L_{2}$ do not contribute to $N_{2,0}$ and will thus be neglected.

The solution $\phi_{2}$ is given by the following form,

$$
\phi_{2}=\phi_{2}^{\mathrm{ns}}+\sum_{\boldsymbol{k}, s}\left[\frac{\cosh (k(z+H))}{\cosh (k H)} \Phi_{2, \boldsymbol{k}}^{s}(t)+\frac{\sinh (k(z+H))}{\cosh (k H)} \Phi_{2, \boldsymbol{k}}^{\mathrm{si}, s}(t)\right] \mathrm{e}^{\mathrm{i} \boldsymbol{k} \cdot \boldsymbol{x}} .
$$

The non-stationarity term $\phi_{2}^{\text {ns }}$ leads to the action evolution term 2.38 , now assuming $\gamma \approx \eta^{2}$. Following the method used at first order, substitution of $(2.53)$ in the bottom boundary condition 2.51) leads to,

$$
\Phi_{2, \boldsymbol{k}}^{\mathrm{si}, s}(t)=-\sum_{\boldsymbol{k}^{\prime}} \frac{\boldsymbol{k}^{\prime} \cdot \boldsymbol{k}}{k} \frac{\cosh (k H)}{\cosh \left(k^{\prime} H\right)} \Phi_{1, \boldsymbol{k}^{\prime}}^{s}(t) G_{\boldsymbol{k}-\boldsymbol{k}^{\prime}} \mathrm{e}^{\mathrm{i} \boldsymbol{l} \cdot \boldsymbol{U} t} .
$$

After calculations detailed in Appendix B, $\phi_{2}$ yields the following contribution to the wave action,

$$
N_{2,0}(\boldsymbol{k})+N_{0,2}(\boldsymbol{k})=-\frac{\pi t}{2} \int_{0}^{2 \pi} M^{2}\left(\boldsymbol{k}, \boldsymbol{k}^{\prime}\right) F^{B}\left(\boldsymbol{k}-\boldsymbol{k}^{\prime}\right) \frac{N_{0}(\boldsymbol{k})}{\sigma \sigma^{\prime}} \frac{k^{\prime 2}}{k^{\prime} C_{g}^{\prime}+\boldsymbol{k}^{\prime} \cdot \boldsymbol{U}} \mathrm{d} \theta^{\prime}+O(1)
$$

in which $\sigma^{\prime}=\sigma-\boldsymbol{l} \cdot \boldsymbol{U}, \sigma^{\prime 2}=g k^{\prime} \tanh (k H)$, and $C_{g}^{\prime}=\sigma^{\prime}\left(1 / 2+k^{\prime} H / \sinh \left(2 k^{\prime} H\right)\right) / k^{\prime}$.

\subsection{Action and momentum balances}

We shall neglect the first order action contribution $N_{1}$ given by (2.40). The solvability condition imposed on the action spectrum is that $N_{2}$ remains an order $\eta^{2}$ smaller than $N_{0}$ for all times. Thus all secular terms of order $\eta^{2}$ must cancel. Combining (2.38), (2.48), and (2.5.5) gives

$$
-\frac{\mathrm{d} N_{0}(\boldsymbol{k})}{\mathrm{d} t}+\frac{\pi}{2} \int_{0}^{2 \pi} M^{2}\left(\boldsymbol{k}, \boldsymbol{k}^{\prime}\right) F^{B}\left(\boldsymbol{k}-\boldsymbol{k}^{\prime}\right) \frac{N_{0}\left(\boldsymbol{k}^{\prime}\right)-N_{0}(\boldsymbol{k})}{\sigma \sigma^{\prime}} \frac{k^{\prime 2}}{k^{\prime} C_{g}^{\prime}+\boldsymbol{k}^{\prime} \cdot \boldsymbol{U}} \mathrm{d} \theta^{\prime}
$$

Since $N_{2}$ and $N_{1}$ remain small, $N(\boldsymbol{k})=N_{0}(\boldsymbol{k})\left[1+O\left(\eta^{2}\right)\right]$, and one has,

$$
\frac{\mathrm{d} N(\boldsymbol{k})}{\mathrm{d} t}=S_{\text {bscat }}(\boldsymbol{k}),
$$

with the spectral action source term,

$$
S_{\text {bscat }}(\boldsymbol{k})=\frac{\pi}{2} \int_{0}^{2 \pi} \frac{k^{2} M^{2}\left(\boldsymbol{k}, \boldsymbol{k}^{\prime}\right)}{\sigma \sigma^{\prime}\left(k^{\prime} C_{g}^{\prime}+\boldsymbol{k}^{\prime} \cdot \boldsymbol{U}\right)} F^{B}\left(\boldsymbol{k}-\boldsymbol{k}^{\prime}\right)\left[N\left(\boldsymbol{k}^{\prime}\right)-N(\boldsymbol{k})\right] \mathrm{d} \theta^{\prime},
$$

where $\sigma^{\prime}=\sigma+\boldsymbol{l} \cdot \boldsymbol{U}$ and $\boldsymbol{k}=\boldsymbol{k}^{\prime}+\boldsymbol{l}$. This interaction rule was already given by Kirby (1988). The only waves that can interact share the same absolute frequency $\omega=\sigma+\boldsymbol{k} \cdot \boldsymbol{U}=$ $\sigma^{\prime}+\boldsymbol{k}^{\prime} \cdot \boldsymbol{U}$. For a given $\boldsymbol{k}$ and without current, the resonant $\boldsymbol{k}^{\prime}$ and $\boldsymbol{l}$ lie on circles in the wavenumber plane (see AH). The current slightly modifies this geometric property. For $U<<C_{g}$ the circles become ellipses (Appendix C).

For a given value of $\omega$, one may obtain the source term integrated over all directions,

$$
S_{\text {bscat }}(\omega)=\int_{0}^{2 \pi} k S_{\text {bscat }}(\boldsymbol{k}) \frac{\partial k}{\partial \omega} \mathrm{d} \theta
$$


Current effects on scattering of surface gravity waves by bottom topography

$$
\begin{aligned}
& =\int_{0}^{2 \pi} \int_{0}^{2 \pi} \frac{\pi}{2} \frac{k^{2} k^{\prime 2} M^{2}\left(\boldsymbol{k}, \boldsymbol{k}^{\prime}\right) F^{B}\left(\boldsymbol{k}-\boldsymbol{k}^{\prime}\right)}{\sigma \sigma^{\prime}\left(k^{\prime} C_{g}^{\prime}+\boldsymbol{k}^{\prime} \cdot \boldsymbol{U}\right)\left(k C_{g}+\boldsymbol{k} \cdot \boldsymbol{U}\right)}\left[N\left(\boldsymbol{k}^{\prime}\right)-N(\boldsymbol{k})\right] d \theta^{\prime} \mathrm{d} \theta \\
& =\int_{0}^{2 \pi} \int_{0}^{2 \pi} \frac{\pi}{2} \frac{M^{2}\left(\boldsymbol{k}, \boldsymbol{k}^{\prime}\right) F^{B}\left(\boldsymbol{k}-\boldsymbol{k}^{\prime}\right)}{\sigma \sigma^{\prime}}\left[\frac{k^{2} N\left(\omega, \theta^{\prime}\right)}{k C_{g}+\boldsymbol{k} \cdot \boldsymbol{U}}-\frac{k^{\prime 2} N(\omega, \theta)}{k^{\prime} C_{g}^{\prime}+\boldsymbol{k}^{\prime} \cdot \boldsymbol{U}}\right] d \theta^{\prime} \mathrm{d} \theta .
\end{aligned}
$$

This expression is anti-symmetric, multiplied by -1 when $\theta$ and $\theta^{\prime}$ are exchanged. Thus $S_{\text {bscat }}(\omega)$ is a substraction of two equal terms, so that for any bottom and wave spectra $S_{\text {bscat }}(\omega)=0$. In other words, the 'source term' is rather an 'exchange term', and conserves the wave action at each absolute frequency. This conservation is consistent with the general wave action conservation theorem proved by Andrews \& McIntyre (1978), which states that there is no flux of action through an unperturbed boundary (here the bottom). It also appears that $\omega$ and $\theta$ are natural spectral coordinates in which the scattering source term takes a symmetric form. Finally, we may consider the equilibrium spectra that satisfy $S_{\text {bscat }}(\boldsymbol{k})=0$ for all $\boldsymbol{k}$. Without current, an equilibrium exists when either $N(\omega, \theta)$ or $N(\boldsymbol{k})$ is isotropic. With current, the scattering term is uniformly zero if and only if the spectral densities in $\boldsymbol{k}$-space, $N(\boldsymbol{k})$, are uniform along the curves of constant $\omega$.

The source term $S_{\text {bscat }}$ may also be re-written in a form corresponding to that in AH, which now appears much less elegant,

$$
S_{\text {bscat }}(\boldsymbol{k})=\int_{0}^{2 \pi} K\left(k, k^{\prime}, H\right) F^{B}\left(\boldsymbol{k}-\boldsymbol{k}^{\prime}\right)\left[N\left(\boldsymbol{k}^{\prime}\right)-N(\boldsymbol{k})\right] d \theta^{\prime},
$$

with

$$
K\left(k, k^{\prime}, H\right)=\frac{\pi k^{\prime 2} M^{2}\left(k, k^{\prime}\right)}{2 \sigma \sigma^{\prime}\left(k^{\prime} C_{g}^{\prime}+\boldsymbol{k}^{\prime} \cdot \boldsymbol{U}\right)}=\frac{4 \pi \sigma k k^{\prime 3} \cos ^{2}\left(\theta-\theta^{\prime}\right)[1+O(F r)]}{\sinh (2 k H)\left[2 k^{\prime} H+\sinh \left(2 k^{\prime} H\right)\left(1+2 \boldsymbol{k}^{\prime} \cdot \boldsymbol{U} / \sigma^{\prime}\right)\right]} .
$$

One may wonder how large is the current-induced scattering represented by $M_{c}$, our eq. (2.34), compared to the bottom-induced scattering represented by $M_{b}$. Since $\sigma^{\prime}=$ $\sigma+(\boldsymbol{U} \cdot \boldsymbol{l})$, the (a) and (b) terms in the numerator $M_{c}$ almost cancel for small Froude numbers, and the (a)+(b) part is of order $F r^{2}$. Thus $M_{c}$ is generally an order $F r^{2}$ smaller than $M_{b}$. For $\boldsymbol{k}$ and $\boldsymbol{k}^{\prime}$ in opposite directions (i.e. back-scattering), the (a)+(b) part is even smaller, of order $g^{2}(\boldsymbol{U} \cdot \boldsymbol{l})^{3} \boldsymbol{l} \cdot \boldsymbol{k}$, and exactly zero in the long wave limit $l H \ll 1$. Thus, for back-scattering, the numerator in $M_{c}$ is itself of the order of (c), i.e. $g^{2}(\boldsymbol{U} \cdot \boldsymbol{l})^{2} \boldsymbol{k} \cdot \boldsymbol{k}^{\prime}$. Interestingly (c) formally comes from the modulations of the surface elevation $\zeta_{1 c}$ so that the $O\left(F r^{2}\right)$ elevation modulation is at least as important as the $\mathrm{O}(\mathrm{Fr})$ current modulation for this back-scattering situation. In that case, $M_{c}$ is of the order of $M_{b} \cosh (k H) \cosh \left(k^{\prime} H\right)(\boldsymbol{U} \cdot \boldsymbol{l})^{2} /\left[g l \alpha_{l} \cosh (l H)\right]$. The relative magnitudes of $M_{b}$ and $M_{c}$ thus depend on $\operatorname{Fr}(l)=(\boldsymbol{U} \cdot \boldsymbol{l}) /[g l \tanh (l H)]^{1 / 2}$ that appears in $(\boldsymbol{U} \cdot \boldsymbol{l})^{2} /\left(g l \alpha_{l}\right)=$ $\operatorname{Fr}^{2}(l) /\left[\operatorname{Fr}^{2}(l)-1\right]$. This $l$-scale Froude number may be formally close to 1 , and thus $M_{c}$ may be larger than $M_{b}$. However, scattering is limited by blocking as no scattered waves can propagate when $C g^{\prime}<\boldsymbol{U} \cdot \boldsymbol{k}^{\prime} / \boldsymbol{k}^{\prime}$. In the long wave limit, $\operatorname{Fr}(l)=F r$ and for $(1-F r) \ll 1$, one has $M_{c}>M_{b}$. For oblique scattering, the (a)+(b) term may dominate the numerator of $M_{c}$ and the situation is more complex. Nevertheless, for Froude numbers typical of continental shelf situations, say $0<F r<0.4, M_{c}$ may be neglected in most situations since its $O\left(F r^{2}\right)$ correction corresponds to only a few percent of the reflection. Obvious exceptions are cases in which $M_{b}$ is zero, such as when $\boldsymbol{k}$ and $\boldsymbol{k}^{\prime}$ are perpendicular.

Finally, we may also write the evolution equation for the wave pseudo-momentum $\mathbf{M}^{w}=\rho_{w} g \int \boldsymbol{k} N(\boldsymbol{k}) \mathrm{d} \boldsymbol{k}$ (see Andrews \& McIntyre 1978), where $\rho_{w}$ is the density of 
sea water. Introducing now the slow medium and wave field variations given by Kirby (1988), that do not interfere with the scattering process, except by probably reducing the surface-bottom bispectrum $Z$, one obtains an extension of the equation of Phillips (1977)

$$
\frac{\partial M_{\alpha}^{w}}{\partial t}+\frac{\partial}{\partial x_{\beta}}\left[\left(U_{\beta}+C_{g \beta}\right) M_{\alpha}^{w}\right]=-\tau_{\alpha}^{\mathrm{bscat}}-M_{\beta}^{w} \frac{\partial U_{\beta}}{\partial x_{\alpha}}-\frac{M_{\alpha}^{w}}{k_{\alpha}} \frac{k \sigma}{\sinh 2 k D} \frac{\partial D}{\partial x_{\alpha}},
$$

with the dummy indices $\alpha$ and $\beta$ denoting dummy horizontal components, and the scattering stress vector,

$$
\tau^{\text {bscat }}=-\rho_{w} g \int \boldsymbol{k} S_{\text {bscat }} \mathrm{d} \boldsymbol{k} .
$$

This stress has dimensions of force per unit area, and corresponds to a force equal to the the divergence of the wave pseudo-momentum flux. Based on the results of LonguetHiggins (1967) and Hara \& Mei (1987), this force does not contribute to the mean flow equilibrium with a balance of the radiation stresses divergence by long waves (or wave setup in stationary conditions), contrary to the initial proposition of Mei (1985). This force is thus a net flux of momentum through the bottom, arising from a correlation between the non-hydrostatic bottom pressure and the bottom slope. That force is likely related to the pressure under partial standing waves locked in phase with the bottom undulations. Although the part $M_{c}$ of the coupling coefficient $M$ given by 2.47 ) is formally due to scattering by the current modulations $\nabla \phi_{1 c}$, and associated surface fluctuations $\zeta_{1 c}$, it should be noted that these motions and related pressures are correlated with the bottom slope in the same way as the part represented by $M_{b}$. Thus both terms contribute to this force $\tau^{\text {bscat }}$ which acts on the bottom and not on the mean flow.

\section{Wave scattering in two dimensions}

Before considering the full complexity of the $3 \mathrm{D}$ wave-bottom scattering in the presence of a current, we first examine the behaviour of the source term in the case of $2 \mathrm{D}$ sinusoidal seabeds. Although the bottom spectrum is not continuous along the $y$-axis, continuity in $x$ is sufficient for the use of (2.45) and the source term can be applied, after proper transformation to remove these singularities. MAHR have investigated the applicability limits of the source term with $U=0$. They proved that for small bottom amplitudes the source term yields accurate reflection estimates, even for localized scatterers, and verified this with test cases. It is thus expected that this also holds for $U \neq 0$.

\subsection{Wave evolution equation in $2 D$}

We consider here a steady wave field in two dimension with incident and reflected waves propagating along the $x$-axis. We shall consider in particular the case of $m$ sinusoidal bars of amplitude $b$ and height $2 b$, with a wavelength $2 \pi / l_{0}$. The bottom elevation is thus

$$
\begin{aligned}
& h(x)=b \sin \left(m l_{0} x\right) \quad \text { for } \quad 0<x<L \\
& h(x)=0 \quad \text { otherwise. }
\end{aligned}
$$

Such a bottom is shown in figure 1 for $m=4$. This form is identical to that of the bottom profile chosen by Kirby (1988) but differs, for $0<x<L$, by a $\pi / 2$ phase shift from the bottom profile chosen by Mei (1985). The bottom spectrum is of the form

$$
F^{B}\left(l_{x}, l_{y}\right)=F^{B 2 D}\left(l_{x}\right) \delta\left(l_{y}\right)
$$


and for the particular bottom given by (3.1),

$$
F^{B 2 D}\left(l_{x}\right)=\left(\frac{1}{2 \pi} \int_{-\infty}^{\infty} h(x) \mathrm{e}^{-\mathrm{i} l x} \mathrm{~d} x\right)^{2}=\frac{2 b^{2} l_{0}^{2}}{\pi L} \frac{\sin ^{2}(l L / 2)}{\left(l_{0}^{2}-l^{2}\right)^{2}}
$$

with

$$
F^{B 2 D}\left( \pm l_{0}\right)=\frac{m b^{2}}{4 l_{0}}=\frac{b^{2} L}{8 \pi}
$$

Note that this is a double-sided spectrum, with only half of the bottom variance contained in the range $l_{x}>0$. For a generic bottom, for which $h(x)$ does not go to zero at infinity, the spectrum is obtained using standard spectral analysis methods, for example, from the Fourier transform of the bottom auto-covariance function (see MAHR). In that case $F^{B 2 D}$ is equivalent to a Wigner distribution (see e.g. Ryzhik et al. 1996).

First, replacing (3.2) in 2.57) removes the angular integral in the source term. Taking $\boldsymbol{k}=\left(k_{x}, k_{y}\right)$, we have $l_{y}=k_{y}-k_{y}^{\prime}=k \sin \theta-k^{\prime} \sin \theta^{\prime}$, thus $\mathrm{d} l_{y}=-k_{y}^{\prime} \cos \theta^{\prime} \mathrm{d} \theta^{\prime}$, and

$$
S_{\text {bscat }}(\boldsymbol{k}, x)=\frac{\pi k^{\prime} M^{2}\left(k, k^{\prime}\right) F^{B 2 D}\left(\boldsymbol{k}-\boldsymbol{k}^{\prime}\right)}{2 \sigma \sigma^{\prime}\left|\cos \theta^{\prime}\right|\left(k^{\prime} C_{g}^{\prime}+\boldsymbol{k}^{\prime} \cdot \boldsymbol{U}\right)}\left[N\left(\boldsymbol{k}^{\prime}\right)-N(\boldsymbol{k})\right] .
$$

Second, assuming now that waves propagate only along the $x$-axis, the wave spectral densities are of the form

$$
N\left(k_{x}, k_{y}\right)=N\left(k_{x}, k_{y}\right) \delta\left(k_{y}\right)=N^{2 D}(k) \delta\left(\theta-\theta_{0}\right) / k,
$$

with $\theta_{0}=0$ for $k_{x}>0$ and $\theta_{0}=\pi$ for $k_{x}<0$. Integrating over $\theta$ removes the singularities on $k_{y}$, and assuming a steady state one obtains

$$
\left[\frac{k_{x}}{k} C_{g}+U_{x}\right] \frac{\partial N^{2 D}}{\partial x}\left(k_{x}, x\right)=S_{\text {bscat }}^{2 D}\left(k_{x}, x\right),
$$

with

$$
S_{\text {bscat }}^{2 D}\left(k_{x}, x\right)=\frac{\pi k^{\prime} M^{2}\left(k, k^{\prime}\right) F^{B 2 D}\left(k_{x}-k_{x}^{\prime}\right)}{2 \sigma \sigma^{\prime}\left(k_{x}^{\prime} C_{g}^{\prime}+k_{x}^{\prime} U_{x}\right)}\left[N^{2 D}\left(k_{x}^{\prime}, x\right)-N^{2 D}\left(k_{x}, x\right)\right] .
$$

Although the present theory is formulated for random waves, there is no possible coupling between waves of different frequencies. Mathematically, it is possible to take the limit to an infinitely narrow wave spectrum, such that, $N^{2 D}(k, x)=N(x) \delta\left(\omega-\omega_{0}\right)+$ $N^{\prime}(x) \delta\left(\omega^{\prime}-\omega_{0}^{\prime}\right)$ with $k_{0 x}>0$ and $k_{0 x}^{\prime}<0$. Using $\partial \omega / \partial k=C_{g}+k_{x} U_{x} /\left|k_{x}\right|$, the resulting evolution equation is, omitting the 0 subscripts on $k$ and $k^{\prime}$,

$$
\begin{aligned}
& {\left[\frac{k_{x}}{k} C_{g}+U_{x}\right] \frac{\partial N}{\partial x}} \\
& =\frac{\pi M^{2}\left(k, k^{\prime}\right) F^{B 2 D}\left(k_{x}-k_{x}^{\prime}\right)}{2 \sigma \sigma^{\prime}}\left[\frac{k N^{\prime}}{k C_{g}+k_{x} U_{x}}-\frac{k^{\prime} N}{k^{\prime} C_{g}^{\prime}+k_{x}^{\prime} U_{x}}\right],
\end{aligned}
$$

with a similar equation for $N^{\prime}$ obtained by exchanging $C_{g}$ and $C_{g}^{\prime}$, and $k^{\prime}$ and $k$, from which it is easy to verify that the total action is conserved.

The stationary evolution equation (3.7) only couples two wave components $N(k)$ and $N\left(k^{\prime}\right)$. For a uniform mean depth $H$, and uniform bottom spectrum $F^{B}$, as considered here, we thus have a linear system of two differential equations, that may be written in 
matrix form for any $k>0$,

$$
\frac{\mathrm{d}}{\mathrm{d} x}\left(\begin{array}{c}
N(k) \\
N\left(k^{\prime}\right)
\end{array}\right)=q Q\left(\begin{array}{c}
N(k) \\
N\left(k^{\prime}\right)
\end{array}\right)
$$

with

$$
q=\frac{\pi M^{2}\left(k, k^{\prime}\right) F^{B 2 D}(l)}{2 \sigma \sigma^{\prime} C g C g^{\prime}}
$$

Defining $l=k_{x}-k_{x}^{\prime}$, the action advection velocities $V^{\prime}=C_{g}^{\prime}+k_{x}^{\prime} U_{x}$ and $V=C_{g}+k_{x} U_{x}$, the terms of the non-dimensional matrix $Q$ are given by

$$
\begin{aligned}
& (Q)_{1,1}=-\frac{C_{g} C_{g}^{\prime}}{V^{2}} \quad \text { and } \quad(Q)_{1,2}=\frac{C_{g} C_{g}^{\prime}}{V V^{\prime}} \\
& (Q)_{2,1}=-\frac{C_{g} C_{g}^{\prime}}{V^{\prime 2}} \quad \text { and } \quad(Q)_{2,2}=\frac{C_{g} C_{g}^{\prime}}{V V^{\prime}}
\end{aligned}
$$

where $(Q)_{i, j}$ is the $i^{\text {th }}$ row and $j^{\text {th }}$ column term of $Q$. The general solution is thus

$$
\left(\begin{array}{c}
N(k, x) \\
N\left(k^{\prime}, x\right)
\end{array}\right)=\mathrm{e}^{q Q x}\left(\begin{array}{c}
N(k, 0) \\
N\left(k^{\prime}, 0\right)
\end{array}\right) .
$$

The matrix exponential is classically the infinite series $\sum_{n=0}^{\infty}(q Q)^{n} / n$ !, in which matrix multiplications are used. The reflection coefficient for the wave action is found using the boundary condition expressing the absence of incoming waves from beyond the bars, $N\left(k^{\prime}, L\right)=0$, giving,

$$
R_{N}=\frac{N\left(k^{\prime}, 0\right)}{N(k, 0)}=-\left(\mathrm{e}^{q Q L}\right)_{2,1} /\left(\mathrm{e}^{q Q L}\right)_{2,2} .
$$

A reflection coefficient for the modulus of the wave amplitude predicted by the source term is thus,

$$
R_{S}=\left[\frac{\sigma^{\prime} N\left(-k^{\prime}, 0\right)}{\sigma N(k, 0)}\right]^{1 / 2}=-\left\{\sigma^{\prime}\left(\mathrm{e}^{q Q L}\right)_{2,1} /\left[\sigma\left(\mathrm{e}^{q Q L}\right)_{2,2}\right]\right\}^{1 / 2}
$$

The spatial variation of the amplitudes may be linear, oscillatory, or exponential, depending on whether the determinant of $Q$, is zero, negative or positive, respectively. That determinant is $C_{g}^{2} C_{g}^{\prime 2}\left(V^{\prime}-V\right)\left(V^{\prime 2}+3 V V^{\prime}+4 V^{4}\right) / V^{4} V^{\prime 3}$, which is always of the sign of $V^{\prime}-V$.

\subsection{Analytical solution for $U=0$}

In the absence of a mean current, $k^{\prime}=-k$, and

$$
(Q)_{1,1}=(Q)_{1,2}=-(Q)_{2,1}=(Q)_{1,1}=1,
$$

Thus $Q^{2}=0$ so that its exponential is only the sum of two terms, $\mathrm{e}^{q Q x}=(I+q Q) x$, where $I$ is the identity matrix. The solution to $(3.9)$ is simply,

$$
\begin{aligned}
N(k, x) & =N(k, 0)\left[\frac{-q(x-L)+1}{1+q L}\right] \\
N(-k, x) & =N(k, 0)\left[\frac{-q(x-L)}{1+q L}\right] .
\end{aligned}
$$

An example of spatial variation of the wave spectrum from $x=0$ to $x=L$ is shown in Figure 2, for $U=0$, and a uniform (white) incident spectrum. The reflected wave energy 

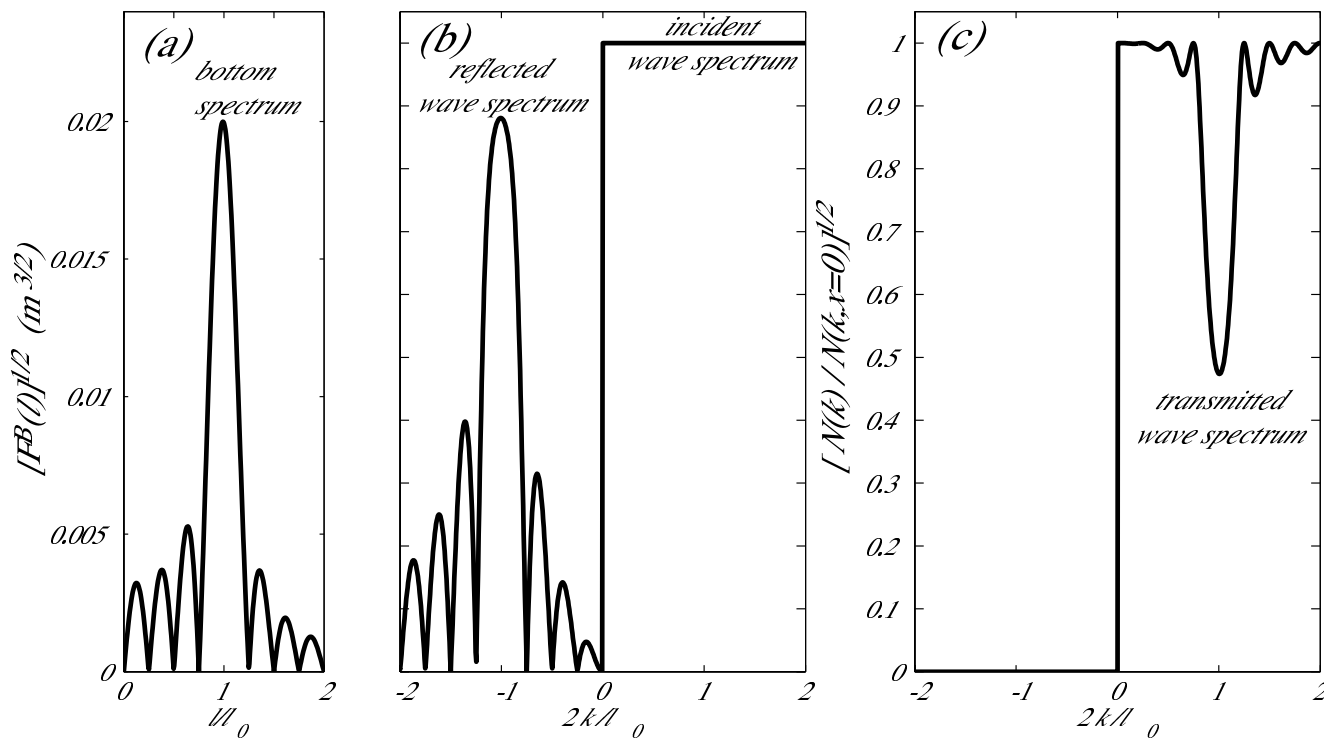

FIGURE 2. Bottom spectrum and evolution of a surface wave spectrum along a field of sinusoidal bars for $U=0, b=0.05 \mathrm{~m}, H=0.156 \mathrm{~m}$, so that $\eta=b / H=0.32$, and $l_{0}=2 \pi, n=4$, so that $L=4 \mathrm{~m}$ (bottom shown in figure 1). (a) square root of the bottom spectrum, (b) and (c) normalized square root wave spectrum upwave (at $x<0)$ and downwave (at $x>L$ ) of the bars, respectively. The incident spectrum $(k>0$ at $x=0)$ is specified to be white (unform in wavenumbers).

(at $k<0$ in figure 2. $a$ ) compensates the loss of energy in the transmitted spectrum (at $k>0$ in figure 2. $\cdot b$ ).

For $k=l / 2$, in the limit of small bar amplitudes, and replacing (3.4) in (3.15) yields

$$
R_{S}=(q L)^{1 / 2}+O(q L)=\frac{k^{2} b L}{2 k H+\sinh (2 k H)}+O(q L)
$$

which is identical to Mei's (1985) equation (3.21)-(3.22) for exact resonance, in the limit of $q L \ll 1$, and also converges to the result of Davies \& Heathershaw (1984) for that same limit. For large bar amplitudes, the reflection is significant if the bars occupy a length $L$ longer than the localization length $1 / q$. However, the reflection coefficient for the wave amplitude only increases with $L$ as $[q L /(1+q L)]^{1 / 2}$, which is slower than the exponential asymptote given by Mei (1985) for sinusoidal bars, and predicted by (Belzons et al. 1988) from the lowest-order theory applied to a random bottom. The present inclusion of the correlations of second-order and zeroth order terms may be thought as the representation of multiple reflections that tend to increase the penetration length in the random medium.

A deeper understanding of this question is provided by the comparison of numerical estimations of the reflection coefficients for the wave amplitudes $R$. A benchmark estimation for linear waves is provided by the step-wise model of Rey (1995) using integral matching conditions for the free propagating waves and three evanescent modes at the step boundaries. This model is known to converge to the reflection coefficents given by an exact solution of Laplace's equation and the boundary conditions, in the limit of an infinite number of steps and evanescent modes. Calculations are performed here with 70 steps and 3 evanescent modes. These numbers are chosen because a larger number of steps or evanescent modes gives indistinguishable results in figure 3. Results of the benchmark model are in good agreement with the measurements of Davies \& Heathershaw (1984), 
except for wave components for which the reflection over the beach, not included in the model, is comparable to the reflection over the bars. An analytical expression $R_{\text {Mei }}$ is given by Mei (1985). $R$ for the present second order theory is given by $R_{S}$ (3.15).

We further compare these estimates to the reflection coefficient $R_{E \text {, Mei }}$ that is deduced from the energy evolution given by Hara \& Mei (1987), using the approximate solutions of Mei (1985, his equations 3.8-3.23). One may prefer to reformulate the energy evolution from the amplitude evolution equations of Kirby (1988) because he used a continuous water depth $h=\sin \left(m l_{0}\right)$, instead of Mei's $h=\cos \left(m l_{0}\right)$ which is discontinuous at $x=0$ and $x=L \dagger$. Yet both Mei's and Kirby's equations lead to the same energy exchange between the incident and reflected components. Using Mei's (1985) notations, the amplitudes of the incident waves, reflected waves, and bottom undulations are $A=$ $2 \sigma \Phi_{0, \boldsymbol{k}}^{+} / g, B=2 \sigma \Phi_{0, \boldsymbol{k}}^{-} / g$, and $D=-2 \mathrm{i} G_{-2 k}$, and the 'cut-off' frequency is

$$
\Omega_{0}=\frac{\sigma k D}{2 \sinh (2 k H)} .
$$

The energy evolution of waves propagating over sinusoidal bars along the $x$-axis is given by Hara \& Mei (1987). The reflected wave energy $B B^{\star} / 2$ should be a solution of

$$
\frac{\partial}{\partial t}\left(\frac{B B^{\star}}{2}\right)-C_{g} \frac{\partial}{\partial x}\left(\frac{B B^{\star}}{2}\right)=\operatorname{Re}\left(\mathrm{i} \Omega_{0} B^{\star} A\right),
$$

where $B^{\star}$ denotes the complex conjugate of $B$. This is identical to 2.39 for a monochromatic bottom except that the imaginary part replaced by a real part.

Equation (3.21) yields a corresponding energy reflection coefficient, given by the fraction of energy lost by the incoming waves,

$$
R_{E, \mathrm{Mei}}=-\frac{1}{C_{g}} \int_{0}^{L} \operatorname{Re}\left(\mathrm{i} \Omega_{0} B^{\star} A\right) \mathrm{d} x .
$$

Simple analytical expressions can be obtained at resonance, where Mei's (1985) eq. (3.20)(3.21) give,

$$
\frac{A B^{\star}}{A^{2}(0)}=\frac{-\mathrm{i} \sinh (2 \tau(1-x / L))}{2 \cosh ^{2} \tau}
$$

with $\tau=\Omega_{0} L / C_{g}$, so that

$$
R_{E, \mathrm{Mei}}=\frac{\cosh 2 \tau-1}{4 \cosh ^{2} \tau}=\frac{1}{2} \tanh ^{2} \tau=\frac{1}{2} R_{\mathrm{Mei}}^{2},
$$

and

$$
R_{E, \text { Mei }}=2^{-1 / 2} R_{\mathrm{Mei}}
$$

It is not surprising that the energy transfer thus computed differs from the energy computed from the amplitude evolution equations. This is typical of small perturbation methods, and was discussed by Hasselmann (1962), among others. Yet, it is remarkable that the ratio of the two is exactly one half. The transfer of energy given by i $\Omega_{0} B^{\star} A$ in (3.21) thus correspond to an amplitude reflection coefficient $R_{E \text {, Mei }}$ that is smaller by a factor $2^{-1 / 2}$, at resonance, compared to $R_{\text {Mei }}$ (figure 3 ). This underprediction of the the reflexion of the energy by (3.24) also has consequences for the analysis and calculation of wave set-up due to wave group propagation over a reflecting bottom. Indeed, the estimation of the scattering stress (2.63), that contribute to the driving of long waves, was

$\dagger$ Such a discontinuous bottom has a markedly different spectrum at low and high frequencies. The present theory, confirmed by calculations with Rey's (1995) numerical model, yield very different reflection coefficients for waves much shorter and much longer than the resonant waves 
analyzed by Hara \& Mei (1987) using a calculation similar to (3.24), which is a factor 2 too small. This may explain, in part, their under-prediction of the observed elevation of the long wave travelling with the incident wave group. However, the present theory, compared to that of Hara \& Mei (1987), is limited to small bar amplitudes, and fails to reproduce their observation of the transition from oscillatory to exponential decay in the spatial evolution of the wave amplitude.

\subsection{Effects of wave and bottom relative phases}

The energy exchange coefficient given by the source term always gives energy to the least energetic components (in the absence of currents), and thus the energy evolution is monotonic. The action source term (2.39) of order $\eta$, that was neglected so far, may have any sign, and thus lead to oscillatory evolutions for the wave amplitudes, as predicted by Mei (1985) and observed by Hara \& Mei (1987). At resonance, and for $U=0$, it can be seen that the first-order energy product $\Phi_{0, k}^{+} \Phi_{0, k}^{-} G_{-2 k}$ in $(2.39)$ is equal to i $A B^{\star} D / 8$, in the limit of a large number of bars. Based on Mei's (1985) approximate solution, in the absence of waves coming from across the bars, this quantity is purely real so that its imaginary part is zero and the corresponding reflection coefficient $R_{S 1}$ is zero. For $U \neq 0$ this property remains as can be seen by replacing Mei's (1985) solution with Kirby's (1988). However, similar correlation terms were also neglected in the second order energy (Appendix B), so that the oscillations of the amplitude across the bar field, observed by Hara and Mei (1987) may occur due to terms of the same order as the scattering source term, including interactions of the sub-harmonic kind (Guazzelli et al. 1992). Further, the bottom-surface bispectrum in $S_{1}$ may become significant if there is a large amount of wave energy coming from beyond the bars. This kind of situation, e.g. due to reflection over a beach, was discussed by Yu \& Mei (2000).

In the absence of such a reflection, and away from resonance but for small values of the scattering strength parameter $\tau=(q L)^{1 / 2}=\Omega_{0} L / C_{g}$, the imaginary part of $\Phi_{0, k}^{+} \Phi_{0, k}^{-} G_{-2 k}$ is an order $(q L)^{1 / 2}$ smaller than the real part and thus contributes a negligible amount to the reflection.

\subsection{Source term and deterministic results for sinusoidal bars}

For large bar amplitudes, such as $\eta=b / H=0.32$ (figure 3.a), all theories with linearized bottom boundary conditions fail to capture the shift of the reflection pattern to lower wavenumbers. This effect was discussed by Rey (1992), and attributed to the non-linear nature of the dispersion relation and the rapid changes in the water depth. Reflection coefficients are still relatively well estimated. For these large amplitudes Mei's (1985) approximate solution is found to be more accurate at resonance compared to the source term. As expected from MAHR and proved here, $R_{\text {Mei }}$ and $R_{S}$ become identical as $\eta=b / H$ goes to zero (figure 3. $b$ ). This fact provides a verification that the first order scattering term $S_{1}$ is different from Hara and Mei's (1987) energy transfer term, and only accounts for a small fraction of the reflection, a fraction that goes to zero as $\eta \rightarrow 0$. It is also found that for all bottom amplitudes, the source term expression provides a simple and accurate solution away from resonance.

Nevertheless, the scattering source term cannot give an accurate description of the spatial variation of the wave amplitude over a deterministic bottom, as shown in figure 4. This is related to the fact that, in MAHR, the present reflection coefficient was obtained from the theory of Pihl et al. (2002) after averaging over the auto-correlation scale of the bottom topography. The present theory can only provide an accurate description of the spatial evolution of the wave field over scales larger than this bottom auto-correlation distance. 


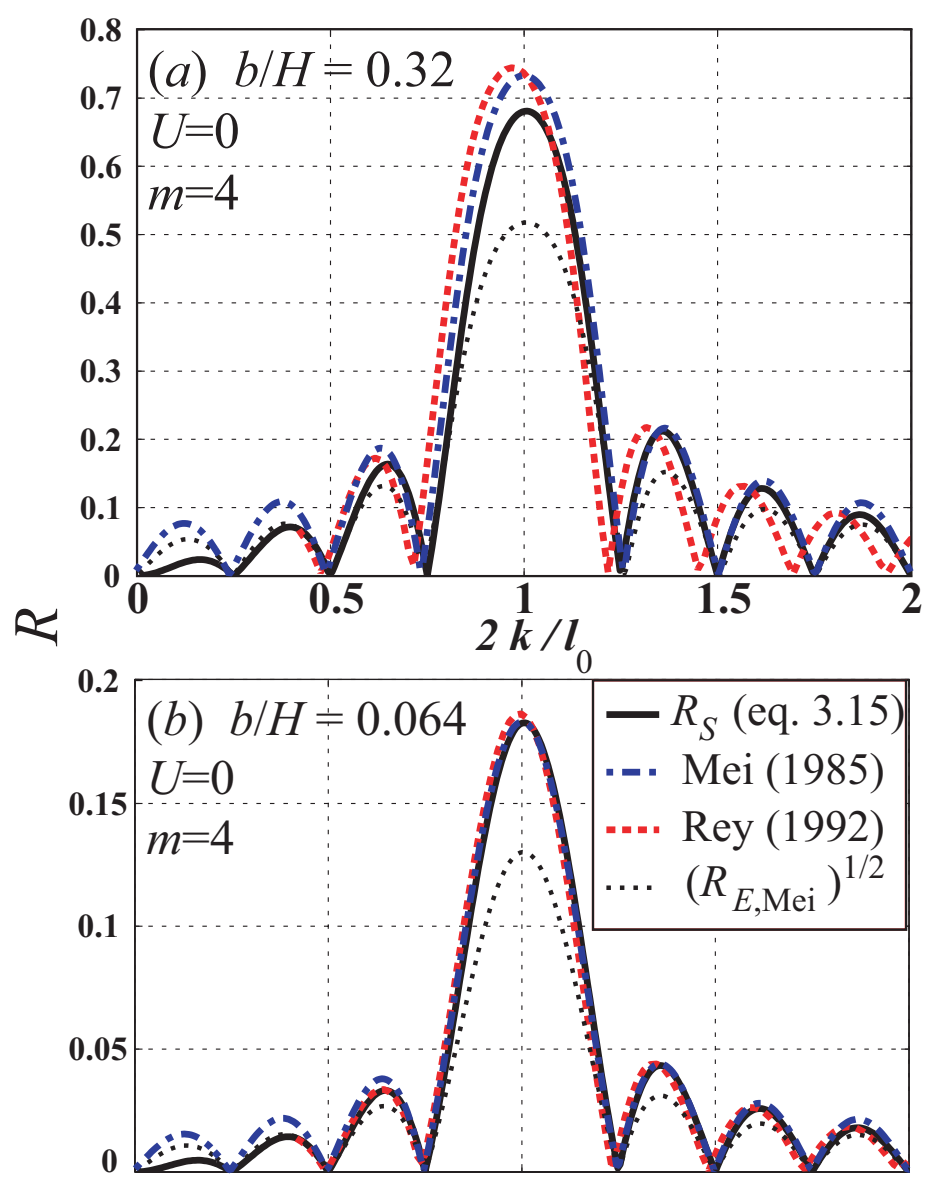

Figure 3. Reflection coefficients for the wave amplitudes for $U=0, H=0.156 \mathrm{~m}, l_{0}=2 \pi$, $n=4$. In (a) $b=0.05$ so that $\eta=b / H=0.32$, corresponding to one of the experiments of Davies \& Heathershaw (1984), and in (b), $b=0.01$, so that $\eta=b / H=0.064$.

\subsection{Effects of currents}

A prominent feature of solutions with current is the modification of the resonant condition from $k=k^{\prime}$ and $l=2 k$, to $\sigma^{\prime}=\sigma+l U$ and $l=k+k^{\prime}$, discussed in detail by Kirby (1988). This shift was verified in the laboratory by Magne, Rey \& Ardhuin (2005). The magnitude of the resonant peak is also largely enhanced for waves against the current, due to a general conservation of the action fluxes and the variation in the action transport velocity, from $C_{g}+U$ for the incident waves, to $C_{g}^{\prime}-U$ for the reflected waves. Further, the modulation of the current and the surface elevation also introduce an additional scattering, via the $M_{c}$ term in the coupling coefficent (2.47). Notations here assume that $\boldsymbol{k}$ is in the direction of the current and $\boldsymbol{k}^{\prime}$ is opposite to the current. At resonance, in the limit $\eta \rightarrow 0$, the amplitude reflection coefficient $R_{S}$ given by (3.15) converges to the reflection coefficient given by Kirby (1988). Using our notations, he obtained

$$
R_{\text {Kirby }}=\left[\frac{\sigma^{\prime}(C g+U)}{\sigma\left(C g^{\prime}-U\right)}\right]^{1 / 2} \tanh (Q L),
$$




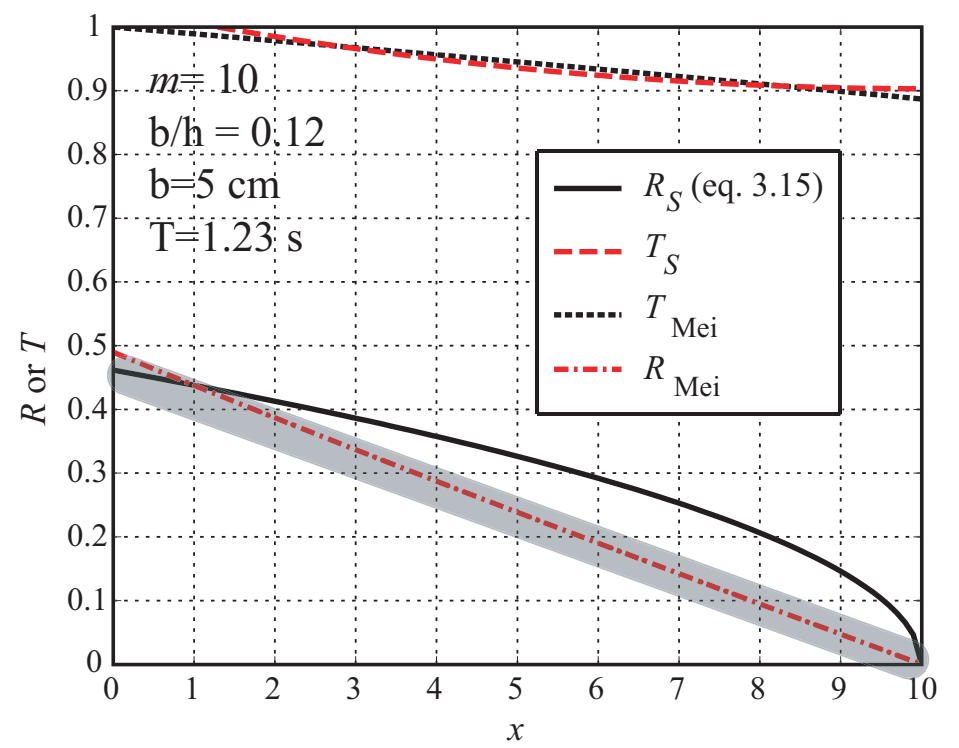

FiguRE 4. Spatial evolution of the incident and reflected wave amplitudes represented by transmission $(T)$ and reflection $(R)$ coefficients, in the near-resonant case $U=0, H=0.156 \mathrm{~m}$, $l_{0}=2 \pi \mathrm{m}^{-1}, m=10$ bars, $b=5 \mathrm{~cm}, \eta=b / H=0.12$ and with a wave period $T=1.23 \mathrm{~s}$. This situation corresponds to one of the experiments of Davies \& Heathershaw (1984), and their measurements lie in the shaded area.

with

$$
Q=\frac{\Omega_{c} \omega}{\left[\sigma \sigma^{\prime}(C g+U)\left(C g^{\prime}-U\right)\right]^{1 / 2}}
$$

and $\Omega_{c}=-M\left(k, k^{\prime}\right) b /\left[4 \omega F^{B}\left(k-k^{\prime}\right)\right]$. Our amplitude reflection coefficient $R_{S}$ is estimated with the approximation $\mathrm{e}^{q Q L}=(I+q Q) L+O\left((q L)^{2}\right)$, so that, to first order in $q L$

$$
R_{S} \approx\left[\frac{\sigma^{\prime} C_{g} C_{g}^{\prime} q L}{\sigma}\right]^{1 / 2} .
$$

Replacing the analytical expression (3.4) in (3.11) yields

$$
R_{S} \approx \frac{b L M\left(k, k^{\prime}\right)}{4\left[\sigma^{2}\left(C_{g}^{\prime}-U\right)^{2}\right]^{1 / 2}},
$$

which is clearly identical to 3.26 at first order in $q L$.

For finite values of $q L$, the reflection coefficient 3.15$)$ corresponding to the solution of (3.9) is obtained by calculating the proper matrix exponential. Anticipating oceanographic conditions with a water depth of $20 \mathrm{~m}$, a strong $2 \mathrm{~m} \mathrm{~s}^{-1}$ current corresponds to a Froude number of 0.17 only. For such a low value of $F r$ in the context of Davies \& Heathershaw's (1984) laboratory experiments, the convergence of the present theory and that of Kirby (1988) is illustrated in figure 5. The reflection coefficient is largely increased for following currents due to the general conservation of the wave action flux. In that case $R$ is enhanced by the factor $\left\{\sigma(C g+U) /\left[\sigma^{\prime}\left(C g^{\prime}-U\right)\right]\right\}^{1 / 2}$. The overall increase in $R$ for following waves amounts to about $60 \%$ at $F r=0.17$, for the laboratory sinusoidal bars of Davies \& Heathershaw (1984) shown before (figure 3), with a reflected wave energy multiplied by a factor 2.5, compared to the case without current. For this mild current 

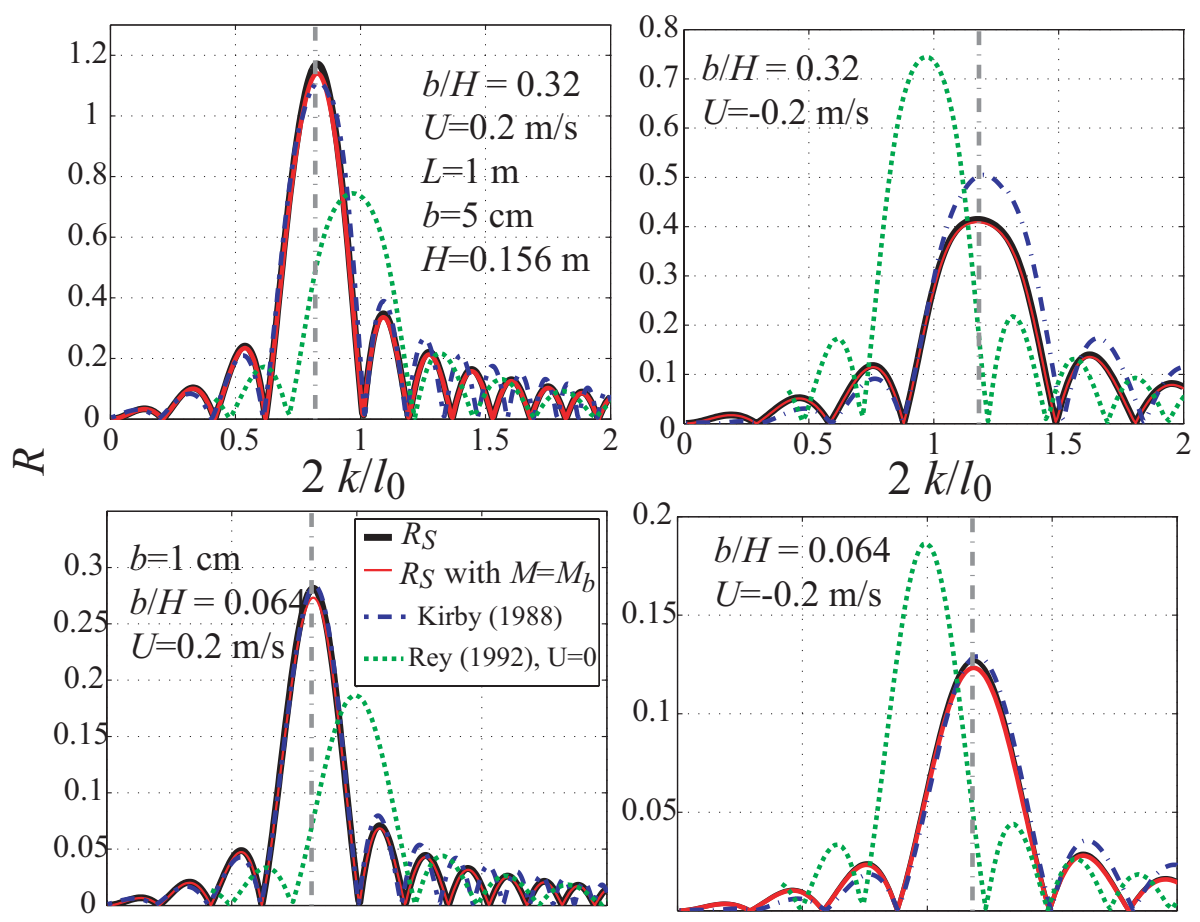

FIGURE 5. Amplitude reflection coefficients for monochromatic waves over sinusoidal bars for the same settings as in figure 3, with a following (left) or opposing (right) current of magnitude $U=0.2 \mathrm{~m} \mathrm{~s}^{-1}$. For reference the reflection coefficient without current, as given by the exact model of Rey (1995), is also shown. The position of the resonant wavenumber is indicated with the grey vertical dash-dotted line.

the contribution of the current fluctuation to the coupling coefficient is small, with a maximum increase of $16 \%$ on the action reflection coefficent, $8 \%$ for the wave amplitude. However, for larger Froude numbers, this additional scattering may become significant as illustrated by figure 6. The present theory and that of Kirby (1988) agree reasonably well for finite values of $\eta$, and we thus expect the source term to represent accurately the scattering of waves over bottom topographies in cases of uniform currents.

For $m=4$ sinusoidal bars, the energy reflection coefficients was found to be within $10 \%$ of the exact solution for over $90 \%$ of the wavenumber range shown in figure 3 , for $\eta<0.1$ and $F r=0$, and this conclusion is expected to hold for $F r<0.2$, given the agreement with Kirby's (1988) approximate solution. This accuracy is twice better than what was found for a rectangular step with $F r=0$ (MAHR). The present method has the advantage of a large economy in computing power. This method is also well adapted for natural sea beds, for which continuous bathymetric coverage is only available in restricted areas, and thus only the statistical properties of the bottom topography are accessible, assuming homogeneity.

\section{Scattering with current on a realistic topography}

\subsection{Sandwaves in the North Sea}

A real ocean topography, at least on the continental shelf, generally presents a continuous and broad bottom elevation spectrum. The effects of a mean current on wave scattering are now examined using a bottom spectrum estimated from a detailed bathymetric survey 


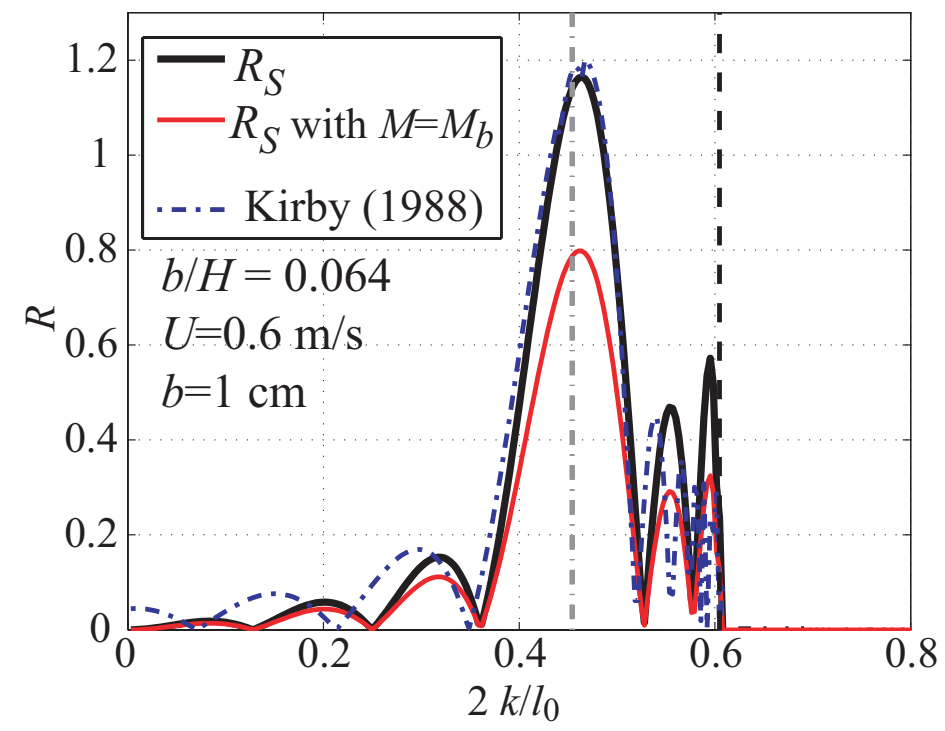

FIGURE 6. Amplitude reflection coefficients for monochromatic waves over sinusoidal bars for the same settings as in figure 3 and 4 , with a stronger following current of magnitude $U=0.6 \mathrm{~m} \mathrm{~s}^{-1}$. The position of the resonant wavenumber is indicated with the grey vertical dash-dotted line. The vertical dashed line corresponds to the wavenumber for which $C g^{\prime}=U$. For larger wavenumbers the reflected waves are blocked and cannot propagate against the current.

of an area centered on the crest of a sand dune, in the southern North Sea (figure 7). In this region, tidal currents are known to generate a wide array of bedforms, from large scale tidal Banks to sand dunes and sand waves (e.g. Dyer \& Huntley 1999; Hulscher \& van den Brink 2001). Although sand dunes present a threat to navigation and are closely monitored (Idier et al. 2002), dunes are much larger than typical wind sea and swell wavelengths. These dunes, however, are generally covered with shorter sandwaves. In the surveyed area the sandwaves have a peak wavelength of $250 \mathrm{~m}$, and an elevation variance of $1.7 \mathrm{~m}^{2}$, which should lead to strong oblique scattering of waves with periods of $10 \mathrm{~s}$ and longer. Over smaller areas of 3 by $3 \mathrm{~km}$ the variance can be as large as $3.3 \mathrm{~m}^{2}$ with a better defined spectral peak, so that our chosen spectrum is expected to be representative of the entire region, including high and low variances on dunes crests and troughs, respectively. The southern North Sea is also known for the attenuation of long swells, generated in the Norwegian Sea. This attenuation has been generally attributed to the dissipation of wave energy by bottom friction (Weber 1991).

The bottom spectrum of the chosen area, like the spectra that were obtained by $\mathrm{AH}$ from the North Carolina shelf, rolls off sharply at high wavenumbers, typically like $l^{-3}$ for the directionally-integrated bottom spectrum $F^{B 2 D}$, and proportional to $l^{-4}$ for the full spectrum $F^{B}$. Here the maximum variance is found for bottom wavelengths of the order of or larger than $250 \mathrm{~m}$ (figure 7). For a typical swell period of $10 \mathrm{~s}$, this corresponds to 2 times the wavelength in $20 \mathrm{~m}$ depth, and thus a rather small scattering angle, $30^{\circ}$ off from the incident direction. Swells propagating from a distant storm, with fixed absolute frequency $\omega=\sigma+\boldsymbol{k} \cdot \boldsymbol{U}$, should be reflected by bottom undulations with widely different variances as the current changes.

Given this bottom spectrum and the mean water depth, simple solutions are available for uniform conditions, because the scattering source term is a linear function of the directional spectrum at a given value of the absolute frequency $\omega$ (see $\mathrm{AH}$ for numerical 

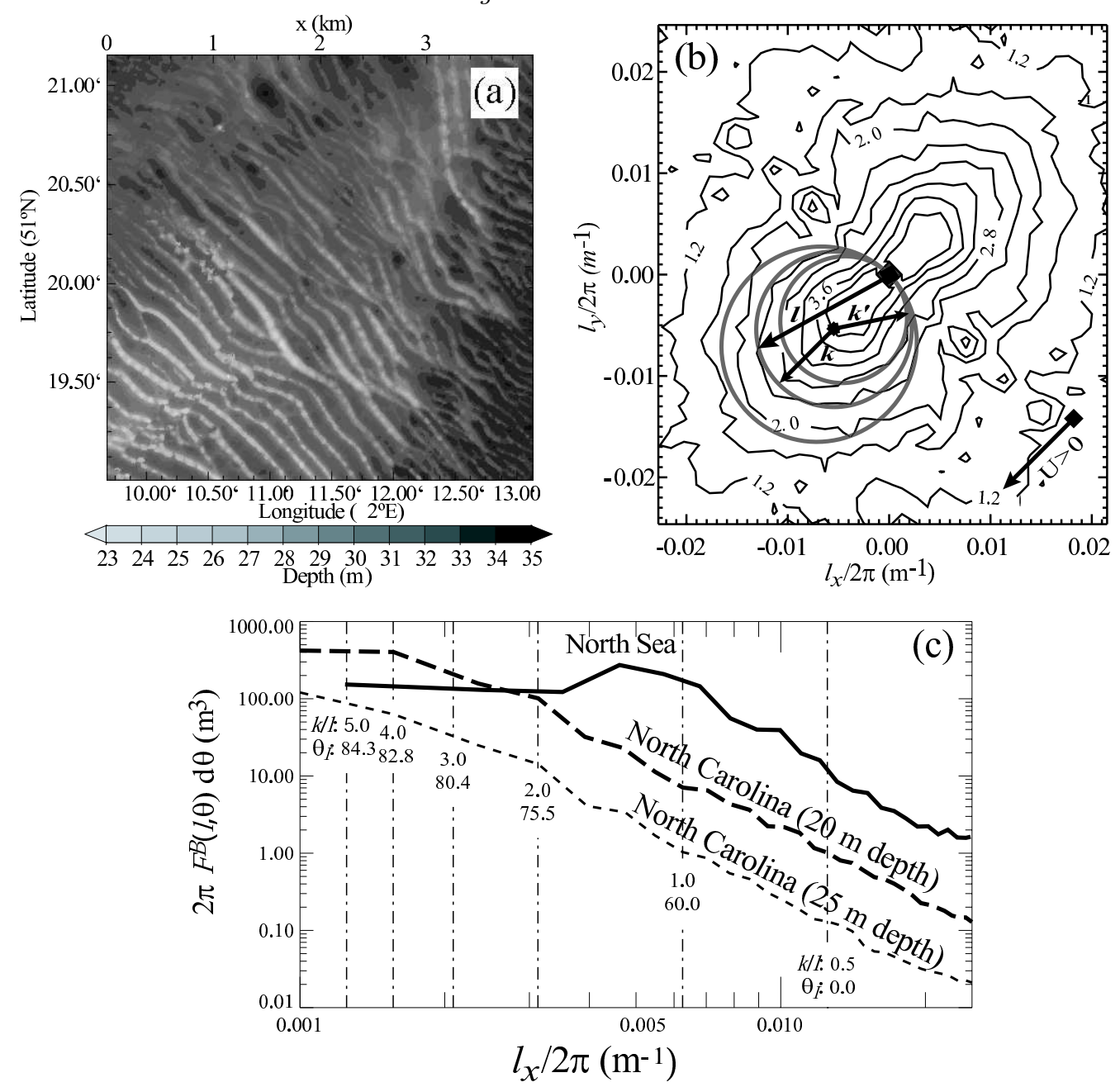

Figure 7. (a) high-resolution bathymetry of a sand wave field in the southern North Sea with depths relative to chart datum, and (b) corresponding bottom elevation spectrum with contour values representing $\log _{10}\left(4 \pi^{2} F^{B}\right)$. The locus of the interacting bottom and surface wave components are indicated for a $12.5 \mathrm{~s}$ waves from the North-East in $25 \mathrm{~m}$ depth, with $U=0$ (middle circle), $U=2 \mathrm{~m} \mathrm{~s}^{-1}$ (smaller ellipse), and $U=-2 \mathrm{~m} \mathrm{~s}^{-1}$ (larger ellipse), $U$ is positive from the North-East. (c) Direction-integrated bottom variance spectra from the North Carolina shelf and the southern North Sea. Vertical lines indicate $k / l$ ratios and incident resonant directions $\theta_{I}$, assuming an incident wave field of $12.5 \mathrm{~s}$ period in $25 \mathrm{~m}$ depth and bedforms parallel to the $y$-axis. For such bedforms, the angle between incident and scattered waves is $180^{\circ}-2 \theta_{I}$.

methods). We consider the wave directional spectrum for a frequency $f_{0}$ and discretize it in $N_{a}$ directions. This spectrum is thus a vector $\mathbf{E}$ in a space with $N_{a}$ dimensions. The square matrix $S$ such that $\mathrm{d} \mathbf{E} / \mathrm{d} t=S \mathbf{E}$ is symmetric and positive, and can thus be diagonalized, which gives $N_{a}$ eigenvalues $\lambda_{n}$ and corresponding eigenvectors $\mathbf{V}_{n}$, such that $\mathbf{S} \mathbf{V}_{n}=\lambda_{n} \mathbf{V}_{n}$. Thus the time evolution is easily obtained by a projection of $\mathbf{E}$ on the basis $\left\{\mathbf{V}_{n}, 1 \leqslant n \leqslant N_{a}\right\}$, giving a decomposition of $\mathbf{E}$ in elementary components. Each of these components of the directional spectrum decays exponentially in time, except for the isotropic part of the spectrum which remains constant because that eigenvector 
corresponds to $\lambda=0$. The eigenvalues thus give interesting timescales for the evolution of the spectrum toward this isotropic state, with a half-life time of each eigenvector given by $-\ln 2 / \lambda_{n}$.

Numerical results are shown here for a mean water depth of $20 \mathrm{~m}$, in order to make the result more visible. For that depth, waves with a period $T=10 \mathrm{~s}$ have a dimensionless depth $k H=1.04$, which is close the value for which the coupling coefficient $M_{b}$ is maximum $(\mathrm{AH})$. As a result, scattering is probably stronger than in real conditions where the mean water depth is $30 \mathrm{~m}$. The following results should still provide some understanding of the likely real effects, at least for larger wave periods with similar values of $k H$. Without current, if $k H$ is kept constant, the magnitude of the coupling coefficient $K\left(k, k^{\prime}, H\right)$ decreases like $H^{-9 / 2}(\mathrm{AH})$, but it is compounded by a higher bottom elevation spectral density for small values of $k$. For back-scattering, the bottom wavenumbers are generally in the range where the bottom spectrum rolls off like $l^{-4}$ (figure 7). Therefore, for these back-scattering directions, the evolution time scale of waves with the same value of $k H$, e.g. $T=11.2 \mathrm{~s}$ in $25 \mathrm{~m}$ depth or $T=13.2 \mathrm{~s}$ in $35 \mathrm{~m}$ depth, is larger by a factor $(25 / 20)^{1 / 2} \simeq 1.1$ or $(35 / 20)^{1 / 2} \simeq 1.3$, respectively. For incident wave and scattering directions for which the bottom spectrum is more uniform and does not compensate for the reduction in the coupling coefficient, such as forward scattering of waves from the North-West, the time scales increase by $(25 / 20)^{9 / 2} \simeq 3.7$ or $(35 / 20)^{9 / 2} \simeq 77$, respectively.

With $N_{a}=120$, corresponding to a directional resolution of $3^{\circ}$, figure 8 shows that the shortest time scales (large negative values of $\lambda_{n}$ ) correspond to directional spectra (eigenvectors) with strong local variations. These eigenvectors are thus associated with scattering at small oblique angles (forward scattering). Only the last 10 eigenvalues have a rather broad support, corresponding to scattering at much larger angles. Besides, the strongest scattering corresponds to a half-life time of $430 \mathrm{~s}$, and mostly affects waves from the North-West or South-East, i.e. propagating in a direction along the sandwave crests. The timescale for waves from the North-East or South-West is about five times larger (the corresponding range of indices is $80<n<110$ ). The $n=118$ eigenvector corresponds to an exchange of wave energy between waves travelling in opposite directions across the sandwaves, but the corresponding half-life is of 3 hours and 15 minutes. Similar results were found for $N_{a}=180$ and $N_{a}=72$ and appear little sensitive to the discretization.

Instead of this idealized horizontally uniform situation, practical situations rather correspond to quasi-stationary conditions with spatial gradients in at least one dimension. In this case the simple steady solutions found above for 2D topography are not physical. Indeed, a 3D bottom causes scattering along the transversal direction $y$, and the energy propagating in that direction builds up slowly up to the point where it becomes as large as the incident wave energy. This process can take a time much longer than the typical duration of a storm or swell arrival, and dissipative processes are likely to be important as the wave energy increases (e.g. Ardhuin et al. 2003). In order to go beyond qualitative statements on time and spatial scales of spectral relaxation, and short of simulating an actual storm in two dimensions, the effects on the wave spectrum are illustrated with a one-dimensional model configuration.

The source term $S_{\text {bscat }}$ was introduced in the version 2.22 of the wave model WAVEWATCH III (Tolman 1991, 2002), based on the wave action evolution equation (2.57) in which the time derivative on the left hand side is now a Lagrangian derivative following a wave packet in physical and spectral space. Bottom scattering is the only source term activated in the present calculation. The model was run with a spectral grid of 30 frequencies ranging from 0.04 to $0.788 \mathrm{~Hz}$ and a directional resolution of $3^{\circ}$. Unfortunately the model spectrum is discretized with components at fixed intrinsic frequencies $\sigma$ and 


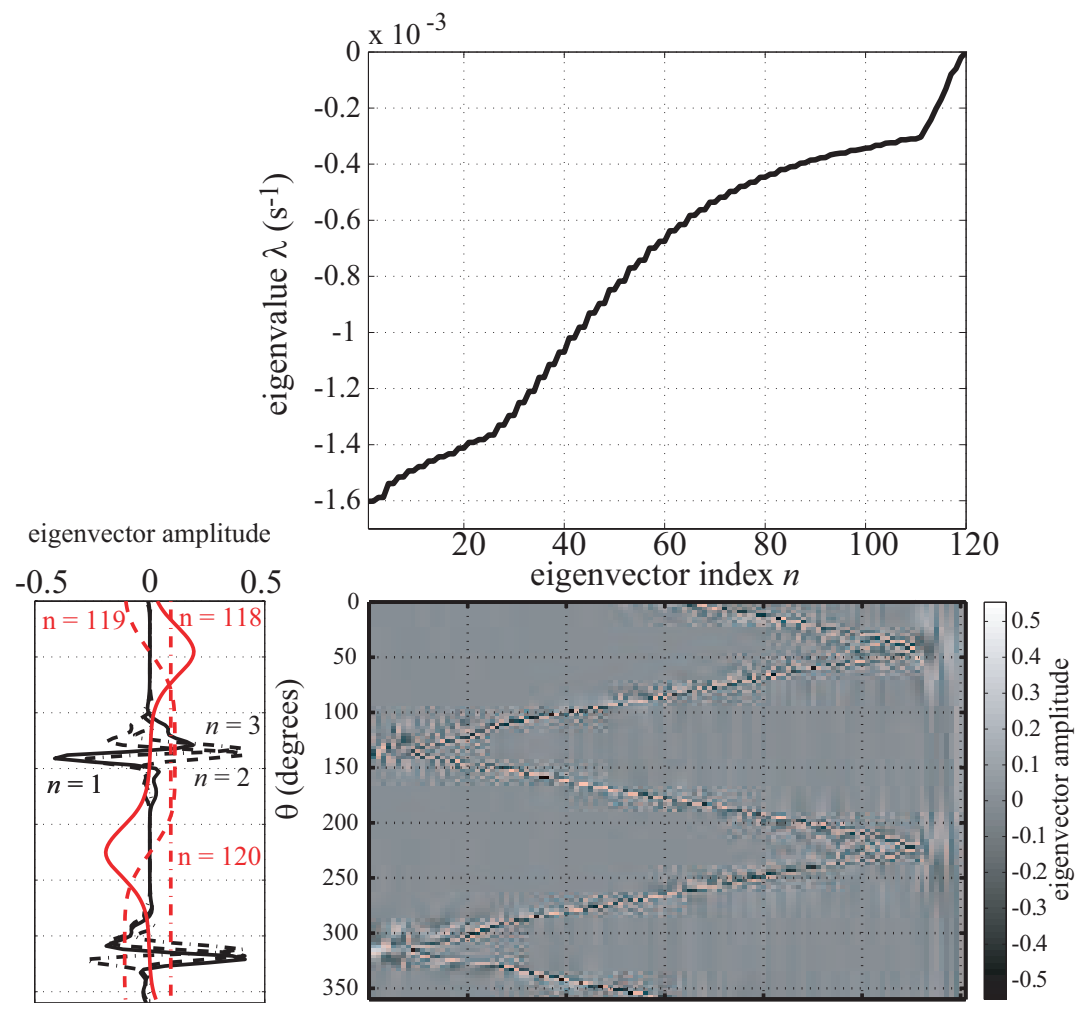

FiguRE 8. Eigenvalues ordered by magnitude (top), and corresponding eigenvectors (bottom right) of the scattering matrix $S$ for $U=0, f_{0}=0.1 \mathrm{~Hz}$, and $H=20 \mathrm{~m}$. The first three and last three eigenvectors are shown in more detail in the bottom left.

directions $\theta$, which is most appropriate for other processes. Therefore a small amount of numerical diffusion leads to a change of action at each absolute frequencies when $U \neq 0$, and the total action is only approximately conserved in that case, with a net change of about $1 \%$ of the integral of the absolute value of the source term for $U= \pm 2 \mathrm{~m} \mathrm{~s}^{-1}$, and four orders of magnitudes smaller, i.e. at the round-off error level, for $U=0$. We have chosen to show cases with significant back-scatter, corresponding to waves normally incident over the sandwaves. This choice also corresponds to a weaker forward scattering, compared to waves propagating along the the sandwave crests.

\subsection{Scattering of waves normally incident on the sandwaves}

To simplify the interpretation of the results, and the processing of the boundary conditions, a one dimensional (East-West) propagation grid is used for the computations, assuming that the wave field, still fully directional, is uniform in the North-South direction. The waves are propagated over a model grid $100 \mathrm{~km}$ long, with a mean depth of $H=20 \mathrm{~m}$, and a spatial grid step of $5 \mathrm{~km}$ (figure 9.a). As discussed above, this water depth is chosen to make the result more visible, and a significant broadening of the incident peak with a (weaker) back-scatter of waves is also found for $H=35 \mathrm{~m}$ and $f_{p}=0.1 \mathrm{~Hz}$ (not shown). A Gaussian incident surface wave spectrum is imposed, with a mean direction from the North-East, a narrow peak directional spread of $12^{\circ}$, and a peak frequency of $0.01 \mathrm{~Hz}$ (figure 9.b). The source term is integrated with a time step of $120 \mathrm{~s}$, and the advection in space uses a third order scheme with a time step of $120 \mathrm{~s}$ (Tolman 2002). 
(a)

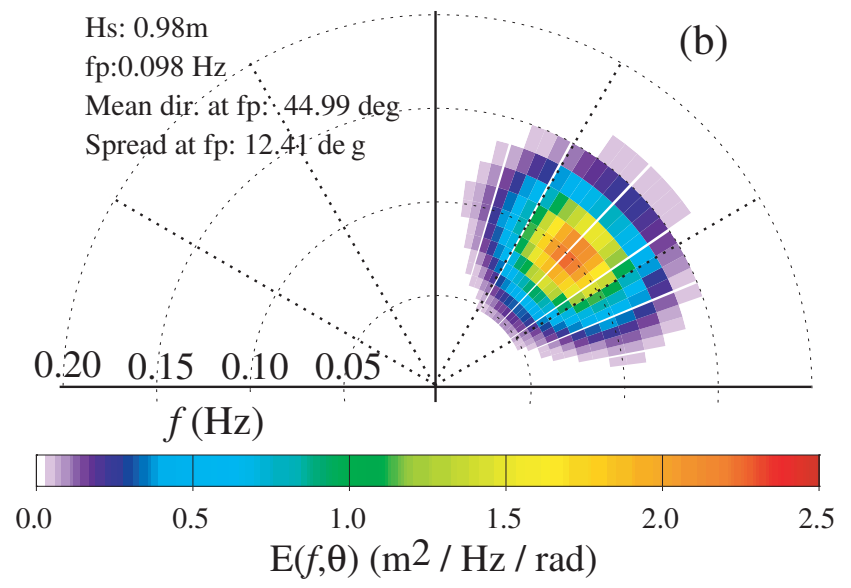

Figure 9. (a) Schematic of the model grid and (b) incident wave spectrum specified at point $F$. Model output is shown below for point $O$. Please note that waves are represented with their arrival direction (direction from, contrary to the standard wind sea convention). The frequency is the relative frequency $\sigma / 2 \pi$.

The scattering source term acts as a diffusion operator with a typical 3-lobe structure, negative at the peak of the wave spectrum, and positive in directions of about $30^{\circ}$ on both sides of the peak. This is identical, but with a larger magnitude, to the effect described by $\mathrm{AH}$. In general the scattering effects are relatively stronger at the lowest frequencies, at least in the range of frequencies used here. For still lower frequencies the scattering coefficient $K$ decreases (see also $\mathrm{AH}$ ) so that, on these spatial scales, very little scattering occurs for infra-gravity waves $(f<0.05 \mathrm{~Hz})$. In addition to this grazingangle forward scattering, a significant back-scatter is found, in particular in the case of following currents.

For an absolute wave frequency of $0.08 \mathrm{~Hz}$, the curves followed by the bottom resonant wavenumbers are overlaid on the bottom spectrum (figure 7 .b). The wavenumbers $\boldsymbol{l}$ along these curves satisfy both the relations $\boldsymbol{k}^{\prime}+\boldsymbol{l}=\boldsymbol{k}$ and $\sigma^{\prime}=\sigma+\boldsymbol{l} \cdot \boldsymbol{U}$. Without current the curve is exactly a circle, and transforms to an ellipse for relatively weak currents (Appendix C). This approximation is used in the model to compute the source term. The current imposed here shifts significantly the resonant configuration for the bottom and surface wavenumbers. A current opposed to the waves enlarges the ellipse towards higher wavenumbers, while a following current will lead to a 'sampling' of shorter wave numbers, i.e. bottom features of larger scales. Since the bottom topography has the largest variance at low wavenumbers, scattering is strongest for following currents (figure 10). With our choice of parameters, there is about a factor 10 reduction in the bottom variance that causes backscatter as $U$ is changed from $2 \mathrm{~m} \mathrm{~s}^{-1}$ to $-2 \mathrm{~m} \mathrm{~s}^{-1}$. Besides, the coupling coefficient $K\left(k^{\prime}, k, H\right)$ is increased in the case of a following current, as discussed above for the $2 \mathrm{D}$ cases.

The resulting wave spectra are also modified due to the conservation of the wave action flux, enhancing the reflected wave energies for $U>0$ (figure 11). This effect is similar to what was found in the $2 \mathrm{D}$ cases considered above, due to the different energy flux velocities $U+C_{g}$ for the incident waves, and $U-C_{g}^{\prime}$ for the reflected waves. In all cases investigated here, the narrow incident wave spectrum is significantly broadened in directions, and that effect is most pronounced for frequencies in the range 0.07-0.10 Hz. Without current or with following currents, spectra in the middle of the model domain 


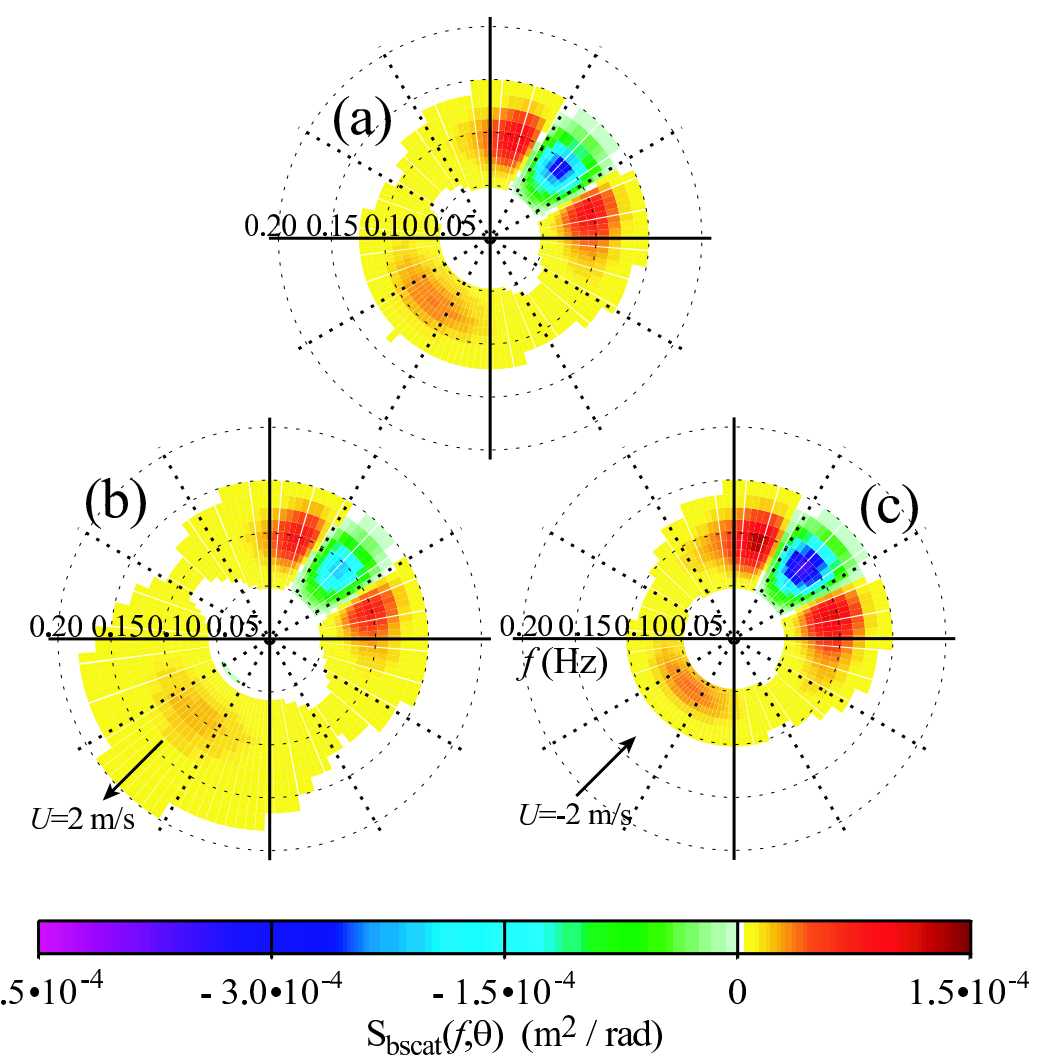

Figure 10. Computed source terms at the boundary forcing point $F$, (a) for $U=0$, (b) for a following current $U=2 \mathrm{~m} \mathrm{~s}^{-1}$, (c) for an opposing current $U=-2 \mathrm{~m} \mathrm{~s}^{-1}$. The frequency is the relative frequency $f=\sigma / 2 \pi$.

exhibit a significant level of back-scattered energy, which increases the significant wave height and the directional spread on the up-wave side of the sandwave field (figure 11). This effect should not be very sensitive to the directional spread of the incident wave field, because the projection of the directional spectrum on the corresponding 'smooth' eigenvectors of the scattering matrix (figure 8) is insensitive to local variations in the directional spectrum. This reflection should thus occur for a wide range of sea states. At the same time, the incident peak of the wave field broadens in directions as it propagates to the down-wave end of the model domain. This broadening is fast close the the forcing boundary (point $\mathrm{F}$ ), with values of the peak frequency directional spreads $\sigma_{\theta, p}$ larger than $35^{\circ}$ at a point $5 \mathrm{~km}$ inside the domain (not shown), and becomes more gradual as the waves propagate, due to the slower evolution of broad spectra that are associated with smaller eigenvalues in the scattering matrix (see also Ardhuin et al. 2003a, Ardhuin $\&$ Herbers 2005). It was also verified that this broadening of the main spectral peak is strongest for waves propagating along the main sandwave crest directions (e.g. from the North-West in our case) due to the larger bottom variance at $\boldsymbol{l}=\boldsymbol{k}-\boldsymbol{k}^{\prime}$ with $\boldsymbol{k} \simeq \boldsymbol{k}^{\prime}$, resulting in a significant modification of the mean direction (Magne 2005).

Finally, a decrease in significant wave height is found along the grid, indicating an attenuation due to wave-bottom scattering. In reality, bottom friction would likely induce a stronger decay, and that decay would be stronger than in the absence of scattering. Essentially the scattering increases the average time taken by wave energy to cross the 


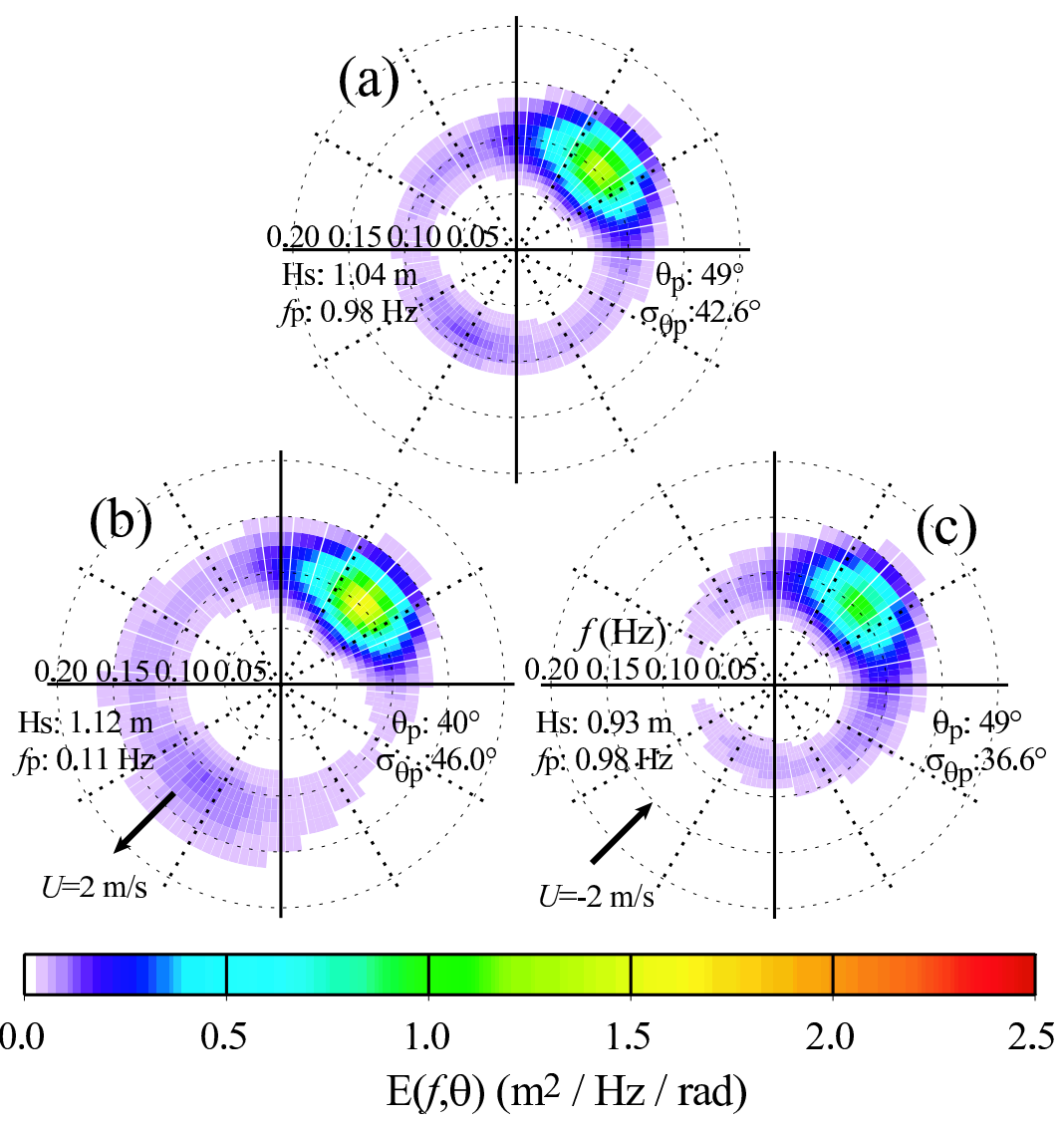

Figure 11. Computed wave spectra at point $O, 40 \mathrm{~km}$ inside of the model domain, after 5 hours of propagation, (a) for $U=0$, (b) for a following current $U=2 \mathrm{~m} \mathrm{~s}^{-1}$, (c) for an opposing current $U=-2 \mathrm{~m} \mathrm{~s}^{-1}$. The frequency is the relative frequency $f=\sigma / 2 \pi$.

domain, and, because of that longer time, bottom friction together with scattering would lead to a larger dissipation than friction alone (Ardhuin et al. 2003).

\section{Conclusion}

The effect of a uniform current on the scattering of random surface gravity waves was investigated theoretically, extending the derivations of Ardhuin \& Herbers (2002). Wave scattering may thus be represented by a scattering source term $S_{\text {bscat }}(\boldsymbol{k})$ for each wave component $\boldsymbol{k}$, in a closed spectral action balance equation. That term gives the rate of exchange of wave action between wave components $\boldsymbol{k}$ and $\boldsymbol{k}^{\prime}$ that have the same absolute frequency, as a result of both water depth variations on the scale of the surface gravity waves wavelength, and current and mean free surface inhomogeneities induced by the bottom topography. The exchange of action between any two wave component pairs $\boldsymbol{k}$ and $\boldsymbol{k}^{\prime}$ is proportional to the bottom elevation spectrum at the wavenumber vector $\boldsymbol{l}=\boldsymbol{k}-\boldsymbol{k}^{\prime}$, which is characteristic of Bragg scattering. The spectral integral of the corresponding wave pseudo-momentum source term $\boldsymbol{k} S_{\text {bscat }}$ gives a recoil force exerted by the bottom on the water column, in addition to the hydrostatic pressure force.

After Magne et al. (2005a) proved that the source term was applicable to non-random topography and accurate in the limit of small bottom amplitudes, just like Bragg scat- 
tering approximations for acoustic or electromagnetic waves (e.g. Elfouhaily \& Guerin 2004), it is further found here that monochromatic wave results are recovered by taking the limit to narrow incident and reflected wave spectra. In absence of current, for a finite sinusoidal bottom and monochromatic waves, the reflection coefficients given by the source term converges to Mei's (1985) theory in the limit of the small bottom amplitudes. The range of maximum reflection and the side lobe pattern of the reflection coefficient as a function of the incident wavenumber is thus a direct consequence of the shape of the bottom spectrum in that case. With this point of view, there is resonance at all wavenumbers but its strength is proportional to the bottom elevation variance at the corresponding scale. In the presence of a current, reflections converge in the same manner to the more general theory of Kirby (1988). In two dimensions, the main effects of a current is an enhancement of reflected wave amplitudes when the incident waves propagate with the current, due to a conservation of the wave action flux, and a Doppler-like shift of the resonant wave frequencies that undergo maximum reflection. The two scale approximation was found to hold very well, even for a relatively fast evolutions of the wave amplitudes over two wavelengths (e.g. figure 3). However, the source term does not give a good representation of the spatial evolution of the wave field on scales shorter that the bottom correlation length, nor can it give reasonable results when another wave train propagates from beyond the bars. In that latter case, a lower order source term must be considered, and a closed action balance cannot be obtained since that extra term depends on the phase relationship between the incident waves, reflected waves and bottom undulations.

In three dimension and over the shallow areas of the southern North Sea, where large sand waves are found with strong tidal currents, wave scattering is expected to be significant, and largely influenced by currents. Over natural topographies, the bottom typically de-correlates over scales shorter than the scattering-induced attenuation scales, so that a modification of the reflection due to a phase locking of the incident and reflected waves with the bottom can be neglected. The wave scattering theory presented in this paper is thus one more piece in the puzzle of wave propagation over shallow continental shelves, and this process may account for a significant part of the observed attenuation of swells in the southern North Sea. The representation of this phenomenon with a source term in the wave action balance equation is expected to be accurate in many conditions of interest. It is consistent with the wide use of phase-averaged models for engineering and scientific purposes when such large scales are involved. The alternative use of phase-resolving elliptic refraction-diffraction models (e.g. Belibassakis et al.2001), is much more expensive in terms of computer resources, due to the necessity to resolve the wave phase and the ellipticity of the problem when back-scattering occurs. For applications to rotational currents, the mean current $U$ should be regarded as the wave advection velocity (Andrews \& McIntyre 1978, see Kirby \& Chen 1989 for practical approximate expressions), but a detailed derivation including scattering by rotational current fluctuations should be the next logical extension of the present theory. This is probably achievable by coupling the rotational part of the flow to the irrotational part, giving a modified Bernoulli equation (e.g. McWilliams et al. 2004). In practice, non-homogeneities in the bottom spectrum will probably have to be addressed due the sharp decrease of the coupling coefficient with water depth, and the generally higher bottom elevation variances in the shallower parts of the sea floor. In particular our limited bathymetric survey shows that sandwaves are modulated by sand dunes, very much like short water waves are modulated by long waves.

This research was supported by a joint grant from CNRS and DGA. Bathymetric data 
Current effects on scattering of surface gravity waves by bottom topography

was acquired by the French Hydrographic and Oceanographic Service (SHOM). Discussions with Michael McIntyre, Kostas Belibassakis, Vincent Rey, and Thierry Garlan and gratefully acknowledged. The results of the relative effects of current modulations and water depths changes owes much to remarks made by anonymous reviewers, without whom the present paper would have been limited to small Froude numbers.

\section{Appendix A. Harmonic oscillator equation for the first order potential}

The harmonic oscillator equation (2.32) can be written as a linear superposition of equations of the type

$$
\frac{d^{2} f_{1}}{d t^{2}}+\omega^{2} f_{1}=\mathrm{e}^{\mathrm{i} \omega^{\prime} t}
$$

In order to specify a unique solution to (A 1), initial conditions must be prescribed. In the limit of the large propagations distances, the initial conditions contribute a negligible non-secular term to the solution. Following Hasselmann (1962), we choose $f_{1}(0)=0$ and $d f_{1} / d t(0)=0$, giving,

$$
\begin{gathered}
f_{1}\left(\omega, \omega^{\prime} ; t\right)=\frac{\mathrm{e}^{\mathrm{i} \omega^{\prime} t}-\mathrm{e}^{\mathrm{i} \omega t}+i\left(\omega-\omega^{\prime}\right) \sin (\omega t) / \omega}{\omega^{2}-\omega^{\prime 2}} \text { for } \omega^{\prime 2} \neq \omega^{2} \\
f_{1}\left(\omega, \omega^{\prime} ; t\right)=\frac{t \mathrm{e}^{\mathrm{i} \omega^{\prime} t}}{2 i \omega^{\prime}}-\frac{\left.\sin ^{\prime} \omega t\right)}{2 i \omega^{\prime} \omega} \text { for } \omega^{\prime}= \pm \omega
\end{gathered}
$$

\section{Appendix B. Harmonic oscillator equation and energy for the second order potential}

Replacing $\phi_{1}(2.30)$ in the surface boundary condition (2.52),

$$
\left(\frac{d^{2}}{d t^{2}}+\sigma^{2}\right) \Phi_{2, \boldsymbol{k}}^{s}(t)=-g k \Phi_{2, \boldsymbol{k}}^{\mathrm{si}, s}-\tanh (k H) \frac{\partial^{2} \Phi_{2, \boldsymbol{k}}^{\mathrm{si}, s}}{\partial t^{2}}+\mathrm{I}-\mathrm{VIII},
$$

and conserving only the resonant terms of $\Phi_{1, \boldsymbol{k}^{\prime}}^{s}$, one obtains

$$
\begin{gathered}
\frac{\partial^{2} \Phi_{2, \boldsymbol{k}}^{\mathrm{si}, s}}{\partial t^{2}}= \\
-\sum_{\boldsymbol{k}^{\prime}, \boldsymbol{k}^{\prime \prime}} \frac{\boldsymbol{k}^{\prime} \cdot \boldsymbol{k}}{k} \frac{\cosh (k H)}{\cosh \left(k^{\prime} H\right)} M\left(\boldsymbol{k}^{\prime}, \boldsymbol{k}^{\prime \prime}\right) G_{\boldsymbol{k}-\boldsymbol{k}^{\prime}} G_{\boldsymbol{k}^{\prime}-\boldsymbol{k}^{\prime \prime}} \Phi_{0, \boldsymbol{k}^{\prime \prime}} \frac{\partial^{2}}{\partial t^{2}}\left(f_{1}\left(\sigma^{\prime}, \boldsymbol{l}^{\prime} \cdot \boldsymbol{U}-s \sigma^{\prime \prime}\right) \mathrm{e} \mathrm{e}^{\mathrm{i} \cdot \boldsymbol{U} t}\right)
\end{gathered}
$$

with $\boldsymbol{l}^{\prime}=\left(\boldsymbol{k}^{\prime \prime}-\boldsymbol{k}^{\prime}\right) \cdot \boldsymbol{U}$. In order to simplify the algebra we assume that the zeroth-order waves are random, with no correlation between $\Phi_{0, \boldsymbol{k}}^{s}$ and $\Phi_{0, \boldsymbol{k}^{\prime \prime}}^{s^{\prime \prime}}$ unless $\boldsymbol{k}= \pm \boldsymbol{k}^{\prime \prime}$ and $s= \pm s^{\prime \prime}$. Thus the only contributing terms to $N_{2,0}$ must verify $\boldsymbol{k}^{\prime \prime}=\boldsymbol{k}$. Only those terms are now written explicitly, the others being grouped in the '...' The amplitude $\Phi_{2, \boldsymbol{k}}^{+}$ satisfies the following forced harmonic oscillator equation,

$$
\left(\frac{\partial^{2}}{\partial t^{2}}+\sigma^{2}\right) \Phi_{2, \boldsymbol{k}}^{+}(t)=\sum_{\boldsymbol{k}^{\prime}} M^{2}\left(\boldsymbol{k}, \boldsymbol{k}^{\prime}\right)\left|G_{\boldsymbol{k}-\boldsymbol{k}^{\prime}}\right|^{2} \Phi_{1, \boldsymbol{k}^{\prime \prime}} f_{1}\left(\sigma^{\prime},-\sigma-\boldsymbol{l} \cdot \boldsymbol{U}\right) \mathrm{e}^{\mathrm{i} \boldsymbol{l} \cdot \boldsymbol{U} t}+\ldots
$$


This is a sum of equations of the form,

$$
\left(\frac{d^{2}}{d t^{2}}+\sigma^{2}\right) f_{2}=f_{1}\left(\sigma^{\prime}, \boldsymbol{l} \cdot \boldsymbol{U}-\sigma ; t\right) \mathrm{e}^{\mathrm{i} l \cdot U t}
$$

The solution $f_{2}$ may be written as

$$
f_{2}=f_{2, a}+f_{2, b}
$$

where

$$
\begin{gathered}
f_{2, a}=-\frac{t \mathrm{e}^{-\mathrm{i} \sigma t}-\sin (\sigma t) / \sigma}{2 \mathrm{i} \sigma\left[\sigma^{\prime 2}-(\boldsymbol{l} \cdot \boldsymbol{U}+\sigma)^{2}\right]} \\
f_{2, b}=-\frac{1}{2 \sigma^{\prime}\left[\sigma^{\prime}-(\boldsymbol{l} \cdot \boldsymbol{U}+\sigma)\right]} \times \\
{\left[\frac{\mathrm{e}^{-\mathrm{i}\left(\sigma^{\prime}-\boldsymbol{l} \cdot \boldsymbol{U}\right) t}}{\sigma^{2}-\left(\sigma^{\prime}-\boldsymbol{l} \cdot \boldsymbol{U}\right)^{2}}-\frac{1}{2 \sigma}\left(\frac{\mathrm{e}^{\mathrm{i} \sigma t}}{\sigma+\left(\sigma^{\prime}-\boldsymbol{l} \cdot \boldsymbol{U}\right)}+\frac{\mathrm{e}^{-\mathrm{i} \sigma t}}{\sigma-\left(\sigma^{\prime}-\boldsymbol{l} \cdot \boldsymbol{U}\right)}\right)\right]}
\end{gathered}
$$

The second order action contribution from correlation between the zeroth and first order velocity potential is given by,

$$
F_{2,0, \boldsymbol{k}}^{\Phi}=F_{0,2, \boldsymbol{k}}^{\Phi}=2\left\langle\Phi_{2, \boldsymbol{k}}^{+} \Phi_{0,-\boldsymbol{k}}^{-}\right\rangle .
$$

This correlation imposes that all non-zero terms must have $\boldsymbol{k}^{\prime \prime}=\boldsymbol{k}$, which removes the '...' terms, so that (B3) becomes

$$
\frac{F_{2,0, \boldsymbol{k}}^{\Phi}}{\Delta \boldsymbol{k}}=2 \sum_{\boldsymbol{k}^{\prime}} M^{2}\left(\boldsymbol{k}, \boldsymbol{k}^{\prime}\right) \frac{\left\langle\left|G_{\boldsymbol{k}-\boldsymbol{k}^{\prime}}\right|^{2}\right\rangle}{\Delta \boldsymbol{k}} \frac{\left\langle\Phi_{0, \boldsymbol{k}}^{+} \Phi_{0,-\boldsymbol{k}}^{-}\right\rangle}{\Delta \boldsymbol{k}}\left\langle f_{2} \mathrm{e}^{\mathrm{i} \sigma t}\right\rangle \Delta \boldsymbol{k},
$$

with

$$
\left\langle f_{2} \mathrm{e}^{\mathrm{i} \sigma t}\right\rangle=\frac{\pi t}{8 \sigma \sigma^{\prime}}\left\{\delta\left[\sigma^{\prime}-(\sigma-\boldsymbol{l} \cdot \boldsymbol{U})\right]+O(1)\right\} .
$$

Taking the limit when $\Delta \boldsymbol{k} \rightarrow 0$, and neglecting $O(1)$ terms yields

$$
F_{2,0}^{\Phi}(t, \boldsymbol{k})=-\int_{\boldsymbol{k}^{\prime}} \frac{\pi t}{4 \sigma} M^{2}\left(\boldsymbol{k}, \boldsymbol{k}^{\prime}\right) F^{B}\left(\boldsymbol{k}-\boldsymbol{k}^{\prime}\right) \frac{F_{0,0}^{\Phi}(\boldsymbol{k})}{\sigma^{\prime}} \delta\left(\omega^{\prime}-\omega\right) \mathrm{d} \boldsymbol{k}^{\prime} .
$$

Changing the spectral coordinates from $\boldsymbol{k}^{\prime}$ to $\left(\omega^{\prime}, \theta^{\prime}\right)$ allows a simple removal of the singularity,

$$
F_{2,0}^{\Phi}(t, \boldsymbol{k})=-\int_{0}^{2 \pi} \frac{\pi t}{4 \sigma} M^{2}\left(\boldsymbol{k}, \boldsymbol{k}^{\prime}\right) F^{B}\left(\boldsymbol{k}-\boldsymbol{k}^{\prime}\right) \frac{F_{0,0}^{\Phi}(\boldsymbol{k})}{\sigma^{\prime}} \frac{k^{\prime}}{C g^{\prime}+\boldsymbol{k}^{\prime} \cdot \boldsymbol{U} / k^{\prime}} \mathrm{d} \theta^{\prime} .
$$

\section{Appendix C. Resonant wavenumber configuration for $U<<C_{g}$}

Under the assumption $U<<C_{g}$, and for a current in the $x$ direction, the resonant conditions

$$
\sigma^{\prime}-\sigma=l_{x} U \text {, and }
$$

yields the following Taylor expansion to first order in $\sigma^{\prime}-\sigma$,

$$
k^{\prime}-k=\left(k_{x}^{\prime}-k_{x}\right) \frac{U}{C_{g}}+O\left[k\left(\frac{U}{C_{g}}\right)^{2}\right] .
$$


We define, $r=k^{\prime}, r_{0}=k, r \cos \theta=k_{x}^{\prime}$, so that

$$
r=r_{0}+\frac{U}{C_{g}}\left(r_{0} \cos \theta_{0}-r \cos \theta\right),
$$

and thus

$$
r=\frac{P}{1+e \cos \theta}
$$

This is the parametric equation of an ellipse of semi-major axis $a$, semi-minor axis $b$, half the foci distance $c$, and eccentricity $e$, with $P=r_{0}+U / C_{g} r_{0} \cos \theta_{0}=b^{2} / a$, and $e=U / C_{g}=c / a$. The interaction between a surface wave with wavenumber $\boldsymbol{k}^{\prime}$ and a bottom component with wavenumber $\boldsymbol{l}$ excites a surface wave with the sum wavenumber $\boldsymbol{k}=\boldsymbol{k}^{\prime}+\boldsymbol{l}$. For a fixed $\boldsymbol{k}$ and current $U$, in the limit of $U<<C_{g}$ the resonant $\boldsymbol{k}^{\prime}$ and $\boldsymbol{l}$ follow ellipses described by their polar equation $(\overrightarrow{\mathrm{C}} 4)$, that reduce to circles for $U=0$.

\section{REFERENCES}

Andrews, D. G. \& McIntyre, M. E. 1978 On wave action and its relatives. J. Fluid Mech. 89, 647-664, corrigendum: vol. 95, p. 796.

Ardhuin, F. \& Herbers, T. H. C. 2002 Bragg scattering of random surface gravity waves by irregular sea bed topography. J. Fluid Mech. 451, 1-33.

Ardhuin, F. \& Herbers, T. H. C. 2005 Numerical and physical diffusion: Can wave prediction models resolve directional spread? J. Atmos. Ocean Technol. 22 (7), 883-892.

Ardhuin, F., Herbers, T. H. C., O’Reilly, W. C. \& Jessen, P. F. 2003 a Swell transformation across the continental shelf. part II: validation of a spectral energy balance equation. J. Phys. Oceanogr. 33, 1940-1953.

Ardhuin, F., O'Reilly, W. C., Herbers, T. H. C. \& Jessen, P. F. $2003 b$ Swell transformation across the continental shelf. part I: Attenuation and directional broadening. J. Phys. Oceanogr. 33, 1921-1939.

BAL, G. \& ChOU, T. 2002 Capillary-gravity wave transport over spatially random drift. Wave Motion 35, 107-124.

Belibassakis, K. A., Athanassoulis, G. A. \& Gerostathis, T. P. 2001 A coupled-mode model for the refraction-diffraction of linear waves over steep three-dimensional bathymetry. Appl. Ocean Res. 23, 319-336.

Benney, D. J. \& Saffman, P. G. 1966 Nonlinear interactions of random waves in a dispersive medium. Proc. Roy. Soc. Lond. A 289, 301-380.

Dalrymple, R., Knight, R. J. \& Lambiase, J. J. 1978 Bedforms and their hydraulic stability relationships in a tidal environment, bay of Fundy, Canada. Nature 275, 100-104.

Dyer, K. R. \& Huntley, D. A. 1999 The origin, classification and modelling of sand banks and ridges. Continental Shelf Research 19, 12851330.

Elfouhaily, T. M. \& GuÉRin, C.-A. 2004 A critical survey of approximate scattering wave theories from random rough surfaces. Waves in Random Media 14, 1-40.

Elter, J. F. \& Molyneux, J. E. 1972 The long-distance propagation of shallow water waves over an ocean of random depth. J. Fluid Mech. 53, 1-15.

Guazzelli, E., Rey, V. \& Belzons, M. 1992 Higher-order Bragg reflection of gravity surface waves by periodic beds. J. Fluid Mech. 245, 301-317.

HarA, T. \& MEI, C. C. 1987 Bragg scattering of surface waves by periodic bars: theory and experiment. J. Fluid Mech. 178, 221-241.

HASSELmanN, K. 1962 On the non-linear energy transfer in a gravity wave spectrum, part 1: general theory. J. Fluid Mech. 12, 481-501.

Hasselmann, K. 1966 Feynman diagrams and interaction rules of wave-wave scattering processes. Rev. of Geophys. 4 (1), 1-32.

Heathershaw, A. D. 1982 Seabed-wave resonance and sand bar growth. Nature 296, 343-345.

Herbers, T. H. C., Hendrickson, E. J. \& O'Reilly, W. C. 2000 Propagation of swell across a wide continental shelf. J. Geophys. Res. 105 (C8), 19,729-19,737.

Herbers, T. H. C., Orzech, M., Elgar, S. \& Guza, R. T. 2003 Shoaling trans- 
formation of wave-frequency directional spectra. J. Geophys. Res. 108 (C1), 3013, doi:10.1029/2001JC001304.

Hulscher, S. J. M. H. \& van Den Brink, G. M. 2001 Comparison between predicted and observed sand waves and sand banks in the north sea. J. Geophys. Res. 106 (C5), 93279338.

Idier, D., Erhold, A. \& Garlan, T. 2002 Morphodynamique d'une dune sous-marine du détroit du pas de calais. Comptes Rendus Géosciences 334, 1079-1085.

Janssen, P. 2004 The interaction of ocean waves and wind. Cambridge: Cambridge University Press.

Janssen, T. T., Herbers, T. H. C. \& Battjes, J. A. 2006 Generalized evolution equation for nonlinear surface gravity waves over two-dimensional topography. J. Fluid Mech. 552, 393-418.

Kirby, J. T. 1986 A general wave equation for waves over rippled beds. J. Fluid Mech. 162, $171-186$.

KirBY, J. T. 1988 Current effects on resonant reflection of surface water waves by sand bars. J. Fluid Mech. 186, 501-520.

Kirby, J. T. \& Chen, T.-M. 1989 Surface waves on vertically sheared flows: approximate dispersion relations. J. Geophys. Res. 94 (C1), 1013-1027.

Longuet-Higgins, M. S. 1950 A theory of the origin of microseisms. Proc. Roy. Soc. Lond. A 243, $1-35$.

Longuet-Higgins, M. S. 1967 On the wave-induced difference in mean sea level between the two sides of a submerged breakwater. J. Mar. Res. 25, 148-153.

Magne, R. 2005 Réflexion des vagues par une topographie sous-marine. PhD thesis, Université de Toulon et du Var.

Magne, R., Ardhuin, F., Rey, V. \& Herbers, T. H. C. $2005 a$ Topographical scattering of waves: spectral approach. J. of Waterway, Port Coast. Ocean Eng. 131 (6), 311-320, arxiv URL: http://arxiv.org/abs/physics/0504148.

Magne, R., Rey, V. \& ARdhuin, F. $2005 b$ Measurement of wave scattering by topography in the presence of currents. Phys. of Fluids 17.

McWilliams, J. C., Restrepo, J. M. \& Lane, E. M. 2004 An asymptotic theory for the interaction of waves and currents in coastal waters. J. Fluid Mech. 511, 135-178.

MEI, C. C. 1985 Resonant reflection of surface water waves by periodic sandbars. J. Fluid Mech. 152, 315-335.

Onorato, M., Osborne, A. R., Serio, M., Cavaleri, L., Brandini, C. \& Stansberg, C. T. 2005 Observation of strongly non-gaussian statistics for random sea surface gravity waves in wave flume experiments. Physical Review E 70, 067302.

Phillips, O. M. 1977 The dynamics of the upper ocean. London: Cambridge University Press, $336 \mathrm{p}$.

Priestley, M. B. 1981 Spectral analysis and time series. London: Academic Press, 890 p.

RAYleigh, L. 1896 The Theory of Sound, 3rd edn. London: Macmillan.

REY, V. 1992 Propagation and local behaviour of normally incident gravity waves over varying topography. Eur. J. Mech. B/Fluids 11 (2), 213-232.

RyzhiK, L., Papanicolaou, G. \& Keller, J. B. 1996 Transport equations for elastic and other waves in random media. Wave Motion 24, 327-370, corrigendum: vol. 95, p. 796.

Tolman, H. L. 1991 A third generation model for wind on slowly varying, unsteady and inhomogeneous depth and currents. J. Phys. Oceanogr. 21, 766-781.

TOLMAN, H. L. 2002 User manual and system documentation of WAVEWATCH-III version 2.22. Tech. Rep. 222. NOAA/NWS/NCEP/MMAB.

WEBER, N. 1991 Bottom friction for wind sea and swell in extreme depth-limited situations. $J$. Phys. Oceanogr. 21, 149-172.

Yu, J. \& MeI, C. C. 2000 Do longshore bars shelter the shore? J. Fluid Mech. 404, 251-268. 